\title{
Changes in Acidic Soil Chemical Properties and Carbon Dioxide Emission Due to Biochar and Lime Treatments
}

\author{
Mehnaz Mosharrof ${ }^{1,2}$, Md. Kamal Uddin 1,*(D), Shamshuddin Jusop ${ }^{1}$, Muhammad Firdaus Sulaiman ${ }^{1}$, \\ S. M. Shamsuzzaman ${ }^{3}$ and Ahmad Numery Ashfaqul Haque ${ }^{1}$
}

1 Department of Land Management, Faculty of Agriculture, Universiti Putra Malaysia, Serdang 43400, Selangor, Malaysia; mmd.mehnaz@gmail.com (M.M.); shamshud@upm.edu.my (S.J.); muhdfirdaus@upm.edu.my (M.F.S.); numerybau@gmail.com (A.N.A.H.)

2 Soil Resource Development Institute (SRDI), Krishi Khamar Sharak, Dhaka 1215, Bangladesh

3 Divisional Laboratory, Soil Resource Development Institute (SRDI), Krishi Khamar Sharak, Dhaka-1215, Bangladesh; shamsuzzamansm@gmail.com

* Correspondence: mkuddin07@gmail.com; Tel.: +60-014-929-4350

\section{check for} updates

Citation: Mosharrof, M.; Uddin, M..K.; Jusop, S.; Sulaiman, M.F.; Shamsuzzaman, S.M.; Haque, A.N.A. Changes in Acidic Soil Chemical Properties and Carbon Dioxide Emission Due to Biochar and Lime Treatments. Agriculture 2021, 11, 219. https://doi.org/10.3390/agriculture 11030219

Academic Editors: Zakaria Solaiman and Hossain Md Anawar

Received: 29 December 2020

Accepted: 4 March 2021

Published: 7 March 2021

Publisher's Note: MDPI stays neutral with regard to jurisdictional claims in published maps and institutional affiliations.

Copyright: (c) 2021 by the authors. Licensee MDPI, Basel, Switzerland. This article is an open access article distributed under the terms and conditions of the Creative Commons Attribution (CC BY) license (https:// creativecommons.org/licenses/by/ $4.0 /)$.

\begin{abstract}
To mitigate global climate change and simultaneously increase soil productivity, the use of biochar in agriculture can be a modern agro-technology that can help in reducing greenhouse gas emissions, enhancing soil carbon sequestration, and ultimately increasing crop yield. This study aimed to evaluate the effects of biochar and lime application on the chemical properties of acid soil and the emission of $\mathrm{CO}_{2}$. A 60-day incubation study was conducted with eleven treatments (T) in which two different biochar produced from rice husk (RHB) and oil palm empty fruit bunches (EFBB) at two rates (10 and $\left.15 \mathrm{t} \mathrm{ha}^{-1}\right)$ and on three rates of dolomitic limestone $(100 \%, 75 \%$, and $50 \%)$, recommended rate of NPK and a control (no amendment). The result showed that biochar and lime significantly increased soil $\mathrm{pH}$, available $\mathrm{P}$, and decreased exchangeable $\mathrm{Al}$ compared to the control. The $\mathrm{pH}$ increase was $44.02 \%$ compared to the control treatment on day 15 , and the available $\mathrm{P}$ was found to be $22.44 \mathrm{mg} \mathrm{kg}^{-1}$ on day 30 from Treatment 7 (75\% lime $\left.+15 \mathrm{t} \mathrm{ha}^{-1} \mathrm{RHB}\right)$. The cumulative $\mathrm{CO}_{2}$ emission from $\mathrm{T}_{7}$ was $207.40 \mu \mathrm{mol} \mathrm{CO} \mathrm{Cm}^{-2}$ that decreased $139.41 \%$ compared to the control. Our findings conclude that RHB with $75 \%$ lime has more potential than EFBB to increase nutrient availability and reduce the emission of $\mathrm{CO}_{2}$ in acid soil.
\end{abstract}

Keywords: acid soil; biochar; lime; carbon dioxide emission; soil nutrients

\section{Introduction}

In many developing countries, soil acidification is a major problem because of crop production's hindrance by intensive farming systems [1]. Approximately $30 \%$ of ice-free land or 3950 million ha of the world's land consists of acidic soils, and more than $50 \%$ of the world's cultivable lands are under the influence of acidification [2]. Different components could contribute to soil acidity, for example, natural measures, industrial contamination, and farming production [3]. In Malaysia, $72 \%$ of the land area is covered by acidic soils (Oxisols and Ultisols) with $\mathrm{pH}<5$ [4].

Liming is the most prominent and efficient practice to neutralize soil acidity of agricultural soil [5-7]. Besides, lime can increase $\mathrm{Ca}$ and $\mathrm{Mg}$ accessibility in soils [8]. To alleviate soil acidity, lime is broadcasted onto the soil surface or incorporated into the soil via tillage operation. However, under no-tillage conditions, surface application of lime is the most appropriate way because lime applied on the surface does not alter the quality of soil which is proved in many previous types of research works [9]. Application of lime increases soil $\mathrm{pH}$, availability of $\mathrm{P}$, cation exchange capacity (CEC), and base saturation and decreases the concentration of $\mathrm{Al}$ [10]. Adding biochar converted from organic residues is an environmentally sound agronomic practice and acts as an ecological risk-free soil conditioner globally [11]. Biochar is a carbon-rich organic material that had gone through 
the pyrolysis cycle of thermal decomposition of biomass when heated to temperatures, usually in the range of 300 and $1000{ }^{\circ} \mathrm{C}$ under low oxygen concentration [12]. Pyrolysis is a thermochemical strategy utilized for changing biomass into biochar at a temperature going from $350^{\circ} \mathrm{C}$ and $700{ }^{\circ} \mathrm{C}$ in an anaerobic condition. Thermochemical methods include pyrolysis and gasification to produce biochar in the solid phase [13]. Depending on the pyrolysis process and the variety of feedstock used, biochar's physico-chemical properties vary [14]. Biochar is utilized as an amendment that can recoup soil fertility and richness, plant development, improve carbon sequestration just as waste administration, and immobilization of contamination [15]. It can be used to enhance plants' growth and increase the yield of crops by increasing a significant amount of soil carbon [16,17].

Many previous researchers reported that soil infertility and low crop productivity are common phenomena in tropical soil. These are influenced by the low soil $\mathrm{pH}$ and low cation exchange capacity (CEC), insufficient nutrients, $\mathrm{Al}$ and Fe toxicity, structural imbalance, etc. [18-22]. Biochar increases the organic carbon in soil and serves as a $C$ sequester to suppress soil organic decomposition [23]. The application of biochar usually raises soil $\mathrm{pH}$ and enhances the availability of nutrients for plant uptake [24]. The ash content of biochar helped to accelerate the $\mathrm{pH}$ of the soil towards neutral [25]. According to Ch'ng et al. [26], phosphorus availability significantly increased by applying biochar, which was fixed by the high amount of $\mathrm{Al}$ and $\mathrm{Fe}$. The bioavailability of macronutrients like $\mathrm{K}, \mathrm{Ca}, \mathrm{Mg}$, and $\mathrm{P}$ increased by using biochar in the soil [21]. In sandy soil examined by Solaiman et al. [27], the addition of biochar, incorporated with or without poultry manure and chemical fertilizer, decreased the insufficiency of both the macro and micronutrients. Utilizing biochar in a farmed land is seemingly the key answer for conserving carbon in soil and is able to alleviate greenhouse gas emissions [28]. Soil $\mathrm{CO}_{2}$ flux significantly increased by the addition of biochar because the biochar increased the amount of soil organic carbon and enhanced the soil's microbial activities $[29,30]$. On the other hand, soil $\mathrm{CO}_{2}$ flux emission decreased by using biochar due to the slow mineralization and decomposition of organic matter resulting from the decreased bioactivity of soluble organic matter by sorption of organic substrate to biochar [31].

Mostly, acidic soils are amended with liming materials to correct the acidity and optimize nutrient availability. However, the lime application for sustainable agriculture is not economically feasible, especially in developing countries, due to less availability and/or high lime cost. Many researchers found that biochar increased nutrient availability in acid soils. On the contrary, biochar application has also been reported to negatively impact nutrient availability in acid soils. In this regard, biochar incorporated with lime can effectively correct the acidity and increase the availability of nutrients. This strategy is eco-friendly for the environment and might be favorable to mitigate greenhouse gas emissions. Research on the effect of RHB and oil palm EFBB with lime on acid soil properties lacks in Malaysia. Therefore, more investigations are needed to provide more evidence for biochar's effect with lime as a soil amendment. In this context, we hypothesized that biochar incorporated with lime would enhance nutrient availability and reduce $\mathrm{CO}_{2}$ emission. The objective of this study was to determine the effects of applying biochar derived from rice husk or oil palm empty fruit bunches in combination with dolomitic limestone on the chemical properties of acid soil and $\mathrm{CO}_{2}$ emission.

\section{Materials and Methods}

\subsection{Soil Collection and Preparation}

The experiment was carried out using the soil of Bungor Series (Typic Paleudult), taken at the depth from 0-20 cm from Taman Partanian, Universiti Putra Malaysia, Puchong, Selangor ( $2^{\circ} 58^{\prime} 59.7^{\prime \prime} \mathrm{N}$ latitude; $101^{\circ} 38^{\prime} 47.5^{\prime \prime} \mathrm{E}$ longitude). The soil sample was air-dried, crushed, and sieved to $<2 \mathrm{~mm}$ before chemical characterization and before treatment. The physical and chemical properties of the initial soil are shown in Table 1. 
Table 1. Selected physico-chemical properties of initial soil.

\begin{tabular}{cc}
\hline Properties & Soil \\
\hline Textural class & Sandy clay loam \\
$\%$ Sand & 69.27 \\
$\%$ Silt & 2.28 \\
$\%$ Clay & 28.44 \\
pH & 4.61 \\
CEC $\left(\mathrm{cmol}_{\mathrm{c}} \mathrm{kg}^{-1}\right)$ & 5.77 \\
Total C $(\%)$ & 1.41 \\
Total N $(\%)$ & 0.07 \\
Total S $(\%)$ & 0.05 \\
Exchangeable K $\left(\mathrm{cmol}_{\mathrm{c}} \mathrm{kg}^{-1}\right)$ & 0.22 \\
Exchangeable Ca $\left(\mathrm{cmol}_{\mathrm{c}} \mathrm{kg}^{-1}\right)$ & 1.46 \\
Exchangeable Mg $\left(\mathrm{cmol}_{\mathrm{c}} \mathrm{kg}^{-1}\right)$ & 0.42 \\
Exchangeable Al $\left(\mathrm{cmol}_{\mathrm{c}} \mathrm{kg}^{-1}\right)$ & 2.49 \\
Available P $\left(\mathrm{mg} \mathrm{kg}^{-1}\right)$ & 5.21 \\
Extractable Fe $\left(\mathrm{mg} \mathrm{kg}^{-1}\right)$ & 99.44 \\
Extractable Mn $\left(\mathrm{mg} \mathrm{kg}^{-1}\right)$ & 4.64 \\
$\mathrm{NH}_{4}-\mathrm{N}\left(\mathrm{mg} \mathrm{kg}^{-1}\right)$ & 16.41 \\
$\mathrm{NO}_{3}-\mathrm{N}\left(\mathrm{mg} \mathrm{kg}^{-1}\right)$ & 11.37 \\
\hline
\end{tabular}

\subsection{Biochar Collection and Characterization}

Two types of biochar used in this incubation experiment are made from locally available feedstock in Malaysia. Rice husk biochar (RHB) was obtained from Sendi Enterprise (Sungai Burong, Selangor, Malaysia) via pyrolyzing rice husk at $300^{\circ} \mathrm{C}$. The particle size of RHB was $1-3 \mathrm{~mm}$, and moisture content was $6 \%$. Oil palm empty fruit bunches biochar (EFBB) was collected from Parkar Go Green Sdn Bhd (Sri Kenari, Kajang, Malaysia), produced through a slow pyrolysis process using medium thermal condition at $300-350{ }^{\circ} \mathrm{C}$. The particle size of the EFB biochar was $2-5 \mathrm{~mm}$, and the moisture content was $5 \%$. Some selected physical and chemical properties of the RHB and EFBB are shown in Table 2.

Table 2. Selected physical and chemical properties of RHB (rice husk), EFBB (empty fruit bunches), dolomitic limestone, and NPK fertilizers.

\begin{tabular}{ccccc}
\hline Properties & RHB & EFBB & Lime & NPK Fertilizer \\
\hline Moisture Content $(\%)$ & 6 & 5 & - & - \\
Ash Content $(\%)$ & 32.40 & 19.72 & - & - \\
pH & 8.15 & 8.53 & 8.5 & - \\
CEC $\left(\mathrm{cmol}_{\mathrm{c}} \mathrm{kg}^{-1}\right)$ & 48.12 & 57.30 & - & - \\
Total C $(\%)$ & 24.86 & 52.11 & & $46(\%)$ \\
Total N $(\%)$ & 1.13 & 0.38 & - & - \\
Total S $(\%)$ & 0.15 & 0.15 & - & $60(\%)\left(\mathrm{K}_{2} \mathrm{O}\right)$ \\
Exchangeable $\mathrm{K}\left(\mathrm{cmol}_{\mathrm{c}} \mathrm{kg}^{-1}\right)$ & 17.45 & 14.86 & - & - \\
Exchangeable Ca $\left(\mathrm{cmol}_{\mathrm{c}} \mathrm{kg}^{-1}\right)$ & 19.46 & 5.08 & $20.00(\%)$ & - \\
Exchangeable $\mathrm{Mg}\left(\mathrm{cmol}_{\mathrm{c}} \mathrm{kg}^{-1}\right)$ & 13.96 & 34.15 & $11.00(\%)$ & $46(\%)\left(\mathrm{P}_{2} \mathrm{O}_{5}\right)$ \\
Total P $\left(\mathrm{mg} \mathrm{kg}^{-1}\right)$ & 3098.40 & 1898.40 & - & - \\
Extractable Fe $\left(\mathrm{mg} \mathrm{kg}^{-1}\right)$ & 43.06 & 24.51 & - & - \\
Extractable $\mathrm{Mn}\left(\mathrm{mg} \mathrm{kg}^{-1}\right)$ & 23.51 & 10.74 & - &
\end{tabular}

A scanning electron microscope (SEM) at the Microscopic Unit of the Institute of Biosciences (IBS), Universiti Putra Malaysia, was used to examine biochar's internal surface and pore structure. Three major types of SEM equipment were used, BAL-TECB SCD 005 Cool Sputter Coater (BALZERS, city FL, USA) was used for metallization, LEO 1455VP (Oxford instrument and INCA software, London, UK) was used for microstructure analysis, and INCA software was used for the image. The biochar was dried and metalized using the BAL-TECB sputter coater syste to get an ideal conductive surface. After metalizing, the 
samples were magnified by $100 \times$ (upper row) and $1000 \times$ (lower row) and analyzed using LEO 1455VP SEM at $15 \mathrm{kV}$.

\subsection{Experimental Treatment and Design}

An incubation experiment was arranged in a complete randomized design (CRD) with three replicates. The detailed treatments were as follows:

$\mathrm{T}_{1}=$ Control (no treatments and fertilizer)

$\mathrm{T}_{2}=$ Recommended rate of NPK $\left(\mathrm{t} \mathrm{ha}^{-1}\right)$

$\mathrm{T}_{3}=100 \%$ dolomitic limestone

$\mathrm{T}_{4}=75 \%$ dolomitic limestone $+10 \mathrm{tha}^{-1}$ rice husk biochar

$\mathrm{T}_{5}=100 \%$ dolomitic limestone $+10 \mathrm{t} \mathrm{ha}^{-1}$ rice husk biochar

$\mathrm{T}_{6}=50 \%$ dolomitic limestone $+15 \mathrm{tha}^{-1}$ rice husk biochar

$\mathrm{T}_{7}=75 \%$ dolomitic limestone $+15 \mathrm{tha}^{-1}$ rice husk biochar

$\mathrm{T}_{8}=75 \%$ dolomitic limestone $+10 \mathrm{tha}^{-1}$ oil palm empty fruit bunches biochar

$\mathrm{T}_{9}=100 \%$ dolomitic limestone $+10 \mathrm{tha}^{-1}$ oil palm empty fruit bunches biochar

$\mathrm{T}_{10}=50 \%$ dolomitic limestone $+15 \mathrm{tha}^{-1}$ oil palm empty fruit bunches biochar

$\mathrm{T}_{11}=75 \%$ dolomitic limestone $+15 \mathrm{tha}^{-1}$ oil palm empty fruit bunches biochar

Where the recommended rate of fertilizer for maize cultivation was respectively applied in all the treatments in the form of urea $\left(140 \mathrm{~kg} \mathrm{ha}^{-1} \mathrm{~N}\right)$, triple superphosphate (TSP) (100 kg ha $\left.{ }^{-1} \mathrm{P}_{2} \mathrm{O}_{5}\right)$, and muriate of potash (MoP) $\left(120 \mathrm{~kg} \mathrm{ha}^{-1} \mathrm{~K}_{2} \mathrm{O}\right)$ [32]. For $200 \mathrm{~g}$ of soil, urea, TSP, and MoP were used $-0.0217 \mathrm{~g}, 0.0155 \mathrm{~g}$, and $0.0143 \mathrm{~g}$, respectively. Biochar

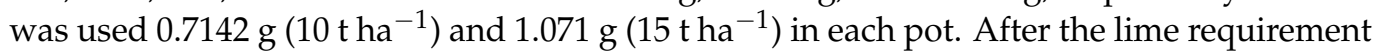
test, the dolomitic limestone was used-0.66 $\mathrm{g}$ (100\%), $0.495 \mathrm{~g}$ (75\%), and $0.33 \mathrm{~g} \mathrm{(50 \% ).}$

\subsection{Incubation Experiment}

The incubation experiment was conducted over 60 days to examine the effects of biochar and lime on soil nutrients. Initially, $200 \mathrm{~g}$ of air-dried soil $(<2 \mathrm{~mm})$ was placed into $500 \mathrm{~mL}$ plastic containers. The moisture content of each sample was adjusted to $24 \%$ of the water holding capacity and readjusted by adding deionized water every 3 days throughout the experiment based on weight loss. During the entire experiment, the temperature was kept constant at $25 \pm 1{ }^{\circ} \mathrm{C}$ in the dark. The soil was sampled on days 15, 30, 45, and 60 for soil $\mathrm{pH}$, total $\mathrm{C}$, total $\mathrm{N}$, available $\mathrm{P}$ exchangeable $\mathrm{K}, \mathrm{Ca}, \mathrm{Mg}$, and $\mathrm{Al}$, extractable $\mathrm{Fe}$ and $\mathrm{Mn}$ content determination. The 60 days incubation experiment was conducted from 17 May to 15 July 2019.

\subsection{Soil and Biochar Analysis}

Soil pH was measured in a 1:2.5 (weight/volume basis) soil:distilled water ratio using glass electrode $\mathrm{pH}$ meter [33], inorganic $\mathrm{N}\left(\mathrm{NH}_{4}{ }^{+}-\mathrm{N}\right.$ and $\left.\mathrm{NO}_{3}{ }^{-}-\mathrm{N}\right)$ was extracted with 1:4 ratio of fresh soil:2 $\mathrm{M} \mathrm{KCl}$ phenylmercuric acetate (KCl-PMA) mixture and determined by titrating against $0.01 \mathrm{~N} \mathrm{HCl}$ [34], total soil carbon (TOC), total nitrogen (TN) and total sulphur (TS) were measured by dry combustion method (Dumas method) using a CNS auto-analyzer (LECO Corporation, St. Joseph, MI, USA) using air dry and ground soil and biochar. The CEC of soil was determined using the ammonium acetate leaching method at $\mathrm{pH}$ 7. The exchangeable cation $\mathrm{Ca}, \mathrm{Mg}$, and $\mathrm{K}$ was extracted with 5:50 ratio of soil:ammonium acetate buffered solution at $\mathrm{pH} 7$ using leaching method where the basic cations adsorbed in soil that replaced by $\mathrm{NH}_{4}{ }^{+}$ion [35] and the concentration was determined by inductively coupled plasma optical emission spectroscopy (ICP-OES, PerkinElmer, Inc., Waltham, MA, USA). Available P was determined by the Bray and Kurtz II method [36], and the concentration was determined by inductively coupled plasma optical emission spectroscopy (ICP-OES, PerkinElmer, Inc., Waltham, MA, USA). Soil exchangeable $\mathrm{Al}$ was extracted using $1 \mathrm{M} \mathrm{KCl}$ and estimated by the titration method [37]. Mehlich No. 1 double acid was used to extract the sample for examining the extractable Fe and $\mathrm{Mn}$, and the concentration was determined using an atomic absorption spectrometer (AAS, PerkinElmer PinAAcle 900T, Waltham, MA, USA) [38]. The bulk density of soil was 
determined by using a core-ring method [38], and a hydrometer was used to determine the soil texture [39].

The $\mathrm{pH}$ of the biochar was measured using a $\mathrm{pH}$ meter by taking a 1:2.5 ratio of air-dried biochar sample to distilled water [40] and electrical conductivity (EC) determined by a conductivity meter at ratio biochar sample to distilled water at 1:10. Total $\mathrm{N}$ and total $C$ in the biochar were analyzed by a CNS analyzer (TruMac CNS Analyser, LECO Corporation, St. Joseph, MI, USA); $1 \mathrm{M} \mathrm{NH}_{4} \mathrm{OAc}$ buffered solution at $\mathrm{pH} 7$ was used for determining biochar $\mathrm{CEC}$ and exchangeable cations [35]. The $\mathrm{K}, \mathrm{Ca}$, and $\mathrm{Mg}$ in the extracts were measured by atomic absorption spectrophotometry (5100 PC, PerkinElmer, Inc., Waltham, MA, USA). For total P determination, the dry ashing method [41] followed by ICP-OES was used. The dry combustion method was used to measure the ash content of biochar. The ash content percentage was calculated as:

$$
\text { Ash content }(\%)=\frac{\text { Weight of ash }(\mathrm{g})}{\text { The dry mass of biochar }(\mathrm{g})} \times 100
$$

where $5.0 \mathrm{~g}$ of each type of biochar sample was taken in a crucible and heated at $500{ }^{\circ} \mathrm{C}$ for $8 \mathrm{~h}$ [42]. After cooling the crucible at room temperature, it was reweighed and calculated by the equation.

\subsection{Percent Relative Data}

The relative data of the value were expressed as percentages, relative to control for each element by the following formula proposed by [43]:

$$
\text { Relative data }(\%)=\frac{\text { Treatment value }- \text { control value }}{\text { control value }} \times 100
$$

where the treatment value was the biochar and lime amended treatment, and the control value was without amendment.

\subsection{Determination of Lime Requirement}

In this study, we used dolomitic limestone. Initially, the amount of dolomitic limestone needed was estimated by a lime requirement test using $10 \mathrm{~g}$ of soil sample with $0.04 \mathrm{~N}$ $\mathrm{Ca}(\mathrm{OH})_{2}$ solution and distilled water. The soil mixture was shaken for $40 \mathrm{~min}$. After that, they were left over $48 \mathrm{~h}$ until the soil $\mathrm{pH}$ reached approximately 7 [44].

\section{8. $\mathrm{CO}_{2}$ Gas Emission Measurement}

Initially, $50 \mathrm{~g}$ of air-dried soil $(<2 \mathrm{~mm})$ was taken into a $500 \mathrm{~mL}$ conical flask with two openings, and the eleven treatments which were mentioned before. The moisture content was adjusted in the previously mentioned way, and all the conical flask was kept in the dark place at $25 \pm 1{ }^{\circ} \mathrm{C} . \mathrm{CO}_{2}$ gas fluxes concentration (in ppm) was measured with three replicates from each treatment, using LI-8100 automated soil $\mathrm{CO}_{2}$ flux system (LI-COR Biosciences, Lincoln, NE, USA) on day 1, 2, 3, 4, 5, 6, 7, 9, 11, 13, 15, 18, 21, 24, 27, 30, 35, $40,45,50,55$ and 60 . At the moment of gas sampling, the conical flux was closed tightly. The inlet and outlet part of the conical flux was attached with two silicon tubes that were connected with LI-COR connectors. By using stop cocks, airflow was controlled that was attached to the silicon tubes. Cumulative gas emissions of $\mathrm{CO}_{2}$ over a 60-day incubation period were calculated by linear interpolation starting from day 1 [45].

\subsection{Statistical Analysis}

All data were analyzed using the analysis of variance (ANOVA) procedure, and means were separated by Tukey's Honestly Significant Difference (HSD) test. Repeated measures analyses were performed on all parameters over time using Statistical Analysis System Software, SAS, version 9.4 (SAS Institute, Cary, NC, USA) $(p \leq 0.05)$. 


\section{Results}

\subsection{SEM Visualization of the Biochars}

The SEM pictures of RHB and EFBB are shown in Figure 1. The permeable structures of each subsequent biochar were found in the SEM micrographs, uncovering an assortment of shapes in the micropores, macropores, also, mesopores. The sample of rice husk biochar has a lot of micropores than EFB biochar. Biochar created from rice husk displayed pores set apart by cell divider structure went from 0.5 to $10 \mu \mathrm{m}$. Oil palm EFBB additionally demonstrated a similar root of pore structure like rice husk, yet the size of the pore went between 1 to $10 \mu \mathrm{m}$. EFB biochar displayed a smoother surface than the RHB.

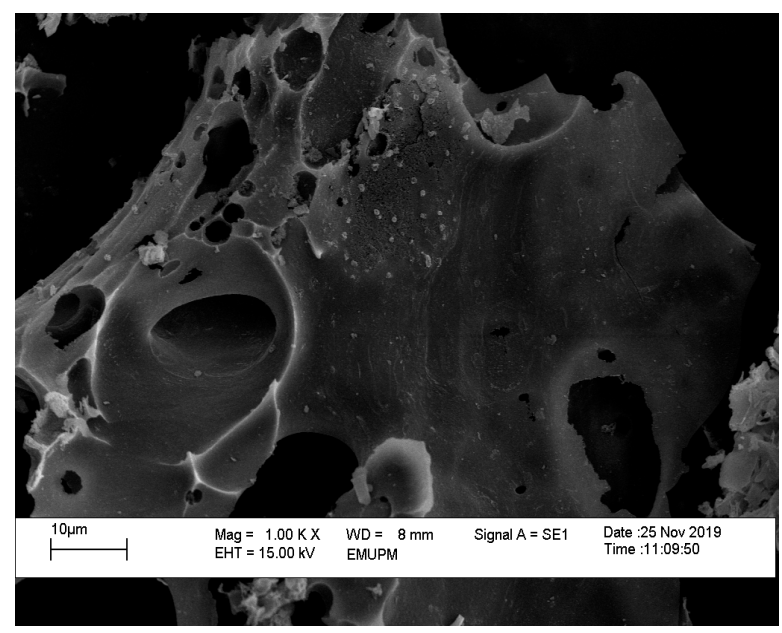

(a)

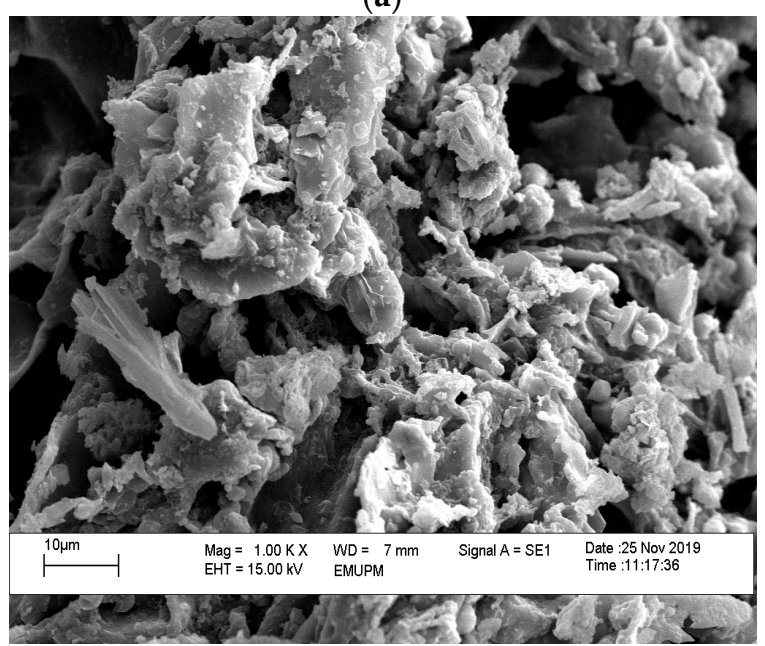

(c)

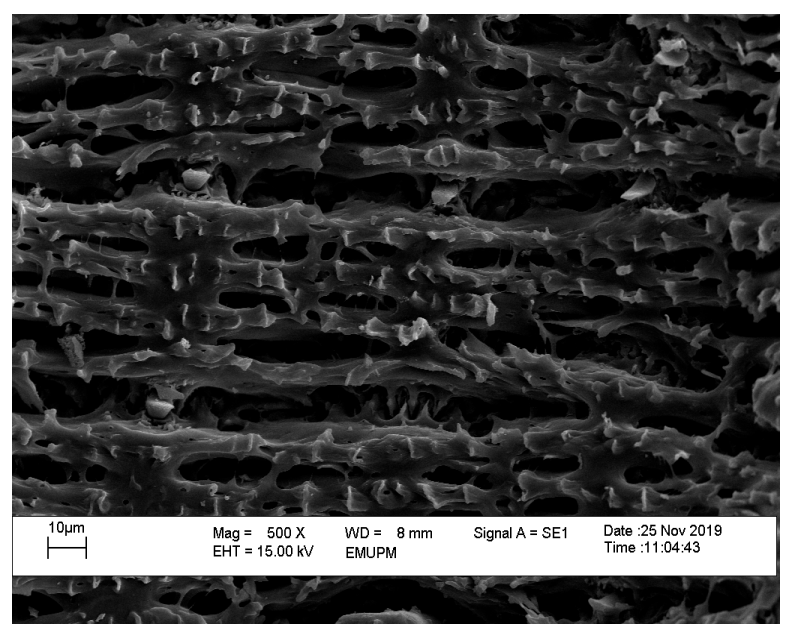

(b)

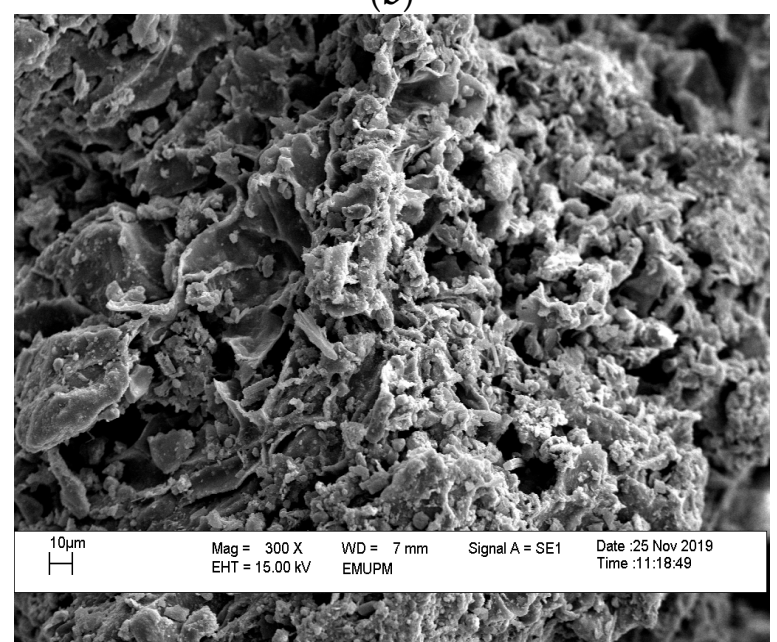

(d)

Figure 1. The micrograph by scanning electron microscopy (SEM) for the RHB (a,b) at $1 \mathrm{~K} \times$ and $500 \times$, and EFBB (c,d) at $1 \mathrm{~K} \times$ and $300 \times$ magnifications. Mag: Magnification, WD: Working distance, SE1: Secondary electron, EHT: Electron high tension, EMUPM: Electron microscopy Universiti Putra Malaysia.

\subsection{Effect of RHB, EFBB, and Dolomitic Limestone Treatments on Soil $p H$}

The repeated measures analysis (Table 3 ) on the soil $\mathrm{pH}$ data illustrated significant interaction effects of treatment combinations and incubation day. Simple effect analysis also indicated that soil $\mathrm{pH}$ was significantly affected by both treatment combinations and duration (day) at each measurement point (Table 4). In general, the highest $\mathrm{pH}$ was observed in $\mathrm{T}_{5}\left(100 \%\right.$ lime $\left.+10 \mathrm{tha}^{-1} \mathrm{RHB}\right)$ at each time of measurement. At 15 days, soil $\mathrm{pH}$ in the $\mathrm{T}_{5}$ was significantly highest (6.53), followed by $\mathrm{T}_{7}$ (75\% lime $\left.+15 \mathrm{tha}^{-1} \mathrm{RHB}\right)$ and $\mathrm{T}_{4}\left(75 \%\right.$ lime $\left.+10 \mathrm{t} \mathrm{ha}^{-1} \mathrm{RHB}\right)$ treatments. After that, it slowly decreased with 
incubation time. The lowest $\mathrm{pH}$ was exhibited by the control $\left(\mathrm{T}_{1}\right)$, followed by $\mathrm{T}_{2}$ and $\mathrm{T}_{6}\left(50 \%\right.$ lime $\left.+15 \mathrm{tha}^{-1} \mathrm{RHB}\right)$ treatments at all interval days. Soil $\mathrm{pH}$ increased with treatment $\mathrm{T}_{5}$ by $2.01 \mathrm{pH}$ unit (6.53-4.52) compared to the control $\left(\mathrm{T}_{1}\right)$.

Table 3. Results of the repeated measures (ANOVA) analysis conducted with Generalized Liner Model(GLM) indicating the $p$ values $/{ }^{*}$ of the treatment, day, and treatment with day interactions of parameters.

\begin{tabular}{|c|c|c|c|c|c|c|}
\hline \multirow[b]{2}{*}{ Factor } & \multicolumn{2}{|c|}{ Treatment } & \multicolumn{2}{|c|}{ Day } & \multicolumn{2}{|c|}{ Treatment ${ }^{*}$ Day } \\
\hline & $p$ Values & $\begin{array}{c}\text { Significant } \\
\text { Level }\end{array}$ & $p$ Values & $\begin{array}{c}\text { Significant } \\
\text { Level }\end{array}$ & $p$ Values & $\begin{array}{c}\text { Significant } \\
\text { Level }\end{array}$ \\
\hline $\mathrm{pH}$ & $<0.0001$ & $* * *$ & $<0.0001$ & $* * *$ & $<0.0001$ & $* * *$ \\
\hline TC & $<0.0001$ & $* * *$ & $<0.0001$ & $* * *$ & 0.0012 & $* *$ \\
\hline TN & 0.0316 & $*$ & 0.0316 & * & 0.4678 & ns \\
\hline $\mathbf{P}$ & $<0.0001$ & $* * *$ & $<0.0001$ & $* * *$ & $<0.0001$ & $* * *$ \\
\hline $\mathbf{K}$ & $<0.0001$ & $* * *$ & $<0.0001$ & $* * *$ & $<0.0001$ & $* * *$ \\
\hline $\mathrm{Ca}$ & $<0.0001$ & $* * *$ & $<0.0001$ & $* * *$ & $<0.0001$ & $* * *$ \\
\hline Mg & $<0.0001$ & $* * *$ & $<0.0001$ & $* * *$ & $<0.0001$ & $* * *$ \\
\hline Al & $<0.0001$ & $* * *$ & $<0.0001$ & $* * *$ & $<0.0001$ & $* * *$ \\
\hline $\mathrm{Fe}$ & $<0.0001$ & $* * *$ & $<0.0001$ & $* * *$ & $<0.0001$ & $* * *$ \\
\hline Mn & $<0.0001$ & $* * *$ & $<0.0001$ & $* * *$ & $<0.0001$ & $* * *$ \\
\hline $\mathrm{CO}_{2}$ & $<0.0001$ & $* * *$ & $<0.0001$ & $* * *$ & $<0.0001$ & $* * *$ \\
\hline
\end{tabular}

Notes: ns (not significant); and ${ }^{*},{ }^{* *}$, and ${ }^{* * *}$, denote significant differences at $p \leq 0.05, p \leq 0.01$, and $p \leq 0.001$, respectively.

Table 4. Effect of RHB, EFBB, and dolomitic limestone treatments on soil $\mathrm{pH}$.

\begin{tabular}{ccccc}
\hline \multirow{2}{*}{ Treatment } & \multicolumn{4}{c}{ Soil pH } \\
\cline { 2 - 5 } & Day 15 & Day 30 & Day 45 & Day 60 \\
\hline $\mathbf{T}_{\mathbf{1}}$ & $4.52 \mathrm{e} \pm 0.023$ & $4.44 \mathrm{~g} \pm 0.020$ & $4.63 \mathrm{e} \pm 0.02$ & $4.51 \mathrm{~h} \pm 0.011$ \\
$\mathbf{T}_{\mathbf{2}}$ & $4.94 \mathrm{~d} \pm 0.050$ & $4.77 \mathrm{f} \pm 0.020$ & $4.69 \mathrm{e} \pm 0.012$ & $4.58 \mathrm{~g} \pm 0.005$ \\
$\mathbf{T}_{\mathbf{3}}$ & $6.28 \mathrm{abc} \pm 0.18$ & $5.80 \mathrm{e} \pm 0.020$ & $5.48 \mathrm{~d} \pm 0.008$ & $5.40 \mathrm{f} \pm 0.008$ \\
$\mathbf{T}_{\mathbf{4}}$ & $6.46 \mathrm{ab} \pm 0.015$ & $6.24 \mathrm{c} \pm 0.17$ & $6.16 \mathrm{~b} \pm 0.015$ & $6.09 \mathrm{bcd} \pm 0.011$ \\
$\mathbf{T}_{\mathbf{5}}$ & $6.53 \mathrm{a} \pm 0.172$ & $6.32 \mathrm{a} \pm 0.020$ & $6.27 \mathrm{a} \pm 0.020$ & $6.21 \mathrm{a} \pm 0.003$ \\
$\mathbf{T}_{\mathbf{6}}$ & $6.09 \mathrm{c} \pm 0.020$ & $5.92 \mathrm{~d} \pm 0.018$ & $5.87 \mathrm{c} \pm 0.023$ & $5.81 \mathrm{e} \pm 0.01$ \\
$\mathbf{T}_{\mathbf{7}}$ & $6.51 \mathrm{a} \pm 0.020$ & $6.31 \mathrm{ab} \pm 0.015$ & $6.21 \mathrm{ab} \pm 0.034$ & $6.12 \mathrm{~b} \pm 0.008$ \\
$\mathbf{T}_{\mathbf{8}}$ & $6.41 \mathrm{ab} \pm 0.019$ & $6.20 \mathrm{c} \pm 0.014$ & $6.14 \mathrm{~b} \pm 0.012$ & $6.05 \mathrm{~d} \pm 0.013$ \\
$\mathbf{T}_{\mathbf{9}}$ & $6.45 \mathrm{ab} \pm 0.020$ & $6.26 \mathrm{abc} \pm 0.015$ & $6.20 \mathrm{ab} \pm 0.015$ & $6.08 \mathrm{~cd} \pm 0.005$ \\
$\mathbf{T}_{\mathbf{1 0}}$ & $6.22 \mathrm{bc} \pm 0.026$ & $5.89 \mathrm{~d} \pm 0.14$ & $5.87 \mathrm{c} \pm 0.011$ & $5.76 \mathrm{e} \pm 0.008$ \\
$\mathbf{T}_{\mathbf{1 1}}$ & $6.42 \mathrm{ab} \pm 0.012$ & $6.24 \mathrm{bc} \pm 0.037$ & $6.23 \mathrm{ab} \pm 0.020$ & $6.12 \mathrm{bc} \pm 0.003$ \\
\hline
\end{tabular}

Means within the same column followed by the same letter are not significantly different at $p \leq 0.05$ (Tukey's HSD test). The column represents the mean values \pm standard error of triplicates.

\subsection{Effect of RHB, EFBB, and Dolomitic Limestone Treatments on Soil Total Carbon}

The repeated measures analysis (Table 3 ) showed that treatment combinations and incubation days significantly interacted with total carbon. The treatment combinations and incubation day also significantly affected total carbon individually (Table 5). The highest value $(2.13 \%)$ was on day 15 , from $\mathrm{T}_{11}\left(75 \%\right.$ lime $\left.+15 \mathrm{t} \mathrm{ha}^{-1} \mathrm{EFBB}\right)$ which is increased $54.35 \%$ compared to the control. After that, it slightly decreased. On the 60th day of incubation, the highest value was increased by $42.25 \%$ from the same treatment compared to the control. 
Table 5. Effect of RHB, EFBB, and dolomitic limestone treatments on soil total carbon.

\begin{tabular}{ccccc}
\hline \multirow{2}{*}{ Treatment } & \multicolumn{4}{c}{ Total Carbon (\%) } \\
\cline { 2 - 5 } & Day 15 & Day 30 & Day 45 & Day 60 \\
\hline $\mathbf{T}_{\mathbf{1}}$ & $1.38 \mathrm{e} \pm 0.017$ & $1.36 \mathrm{~g} \pm 0.018$ & $1.40 \mathrm{~h} \pm 0.018$ & $1.42 \mathrm{~g} \pm 0.034$ \\
$\mathbf{T}_{\mathbf{2}}$ & $1.66 \mathrm{~d} \pm 0.026$ & $1.54 \mathrm{f} \pm 0.029$ & $1.61 \mathrm{~g} \pm 0.03$ & $1.59 \mathrm{f} \pm 0.017$ \\
$\mathbf{T}_{\mathbf{3}}$ & $1.69 \mathrm{fg} \pm 0.033$ & $1.69 \mathrm{e} \pm 0.012$ & $1.71 \mathrm{fg} \pm 0.032$ & $1.61 \mathrm{ef} \pm 0.023$ \\
$\mathbf{T}_{\mathbf{4}}$ & $1.88 \mathrm{c} \pm 0.03$ & $1.71 \mathrm{e} \pm 0.02$ & $1.81 \mathrm{ef} \pm 0.033$ & $1.74 \mathrm{~d} \pm 0.027$ \\
$\mathbf{T}_{\mathbf{5}}$ & $1.97 \mathrm{bc} \pm 0.015$ & $1.73 \mathrm{de} \pm 0.012$ & $1.86 \mathrm{ed} \pm 0.019$ & $1.78 \mathrm{~cd} \pm 0.036$ \\
$\mathbf{T}_{\mathbf{6}}$ & $1.99 \mathrm{bbc} \pm 0.029$ & $1.85 \mathrm{~cd} \pm 0.032$ & $1.93 \mathrm{~cd} \pm 0.014$ & $1.78 \mathrm{~cd} \pm 0.032$ \\
$\mathbf{T}_{\mathbf{7}}$ & $2.01 \mathrm{ab} \pm 0.02$ & $1.91 \mathrm{bc} \pm 0.027$ & $1.96 \mathrm{~cd} \pm 0.015$ & $1.86 \mathrm{~cd} \pm 0.033$ \\
$\mathbf{T}_{\mathbf{8}}$ & $2.05 \mathrm{ab} \pm 0.026$ & $1.93 \mathrm{bc} \pm 0.035$ & $2.01 \mathrm{abc} \pm 0.026$ & $1.91 \mathrm{abc} \pm 0.012$ \\
$\mathbf{T}_{\mathbf{9}}$ & $2.06 \mathrm{ab} \pm 0.0176$ & $1.95 \mathrm{bc} \pm 0.02$ & $2.06 \mathrm{ab} \pm 0.023$ & $1.94 \mathrm{ab} \pm 0.026$ \\
$\mathbf{T}_{\mathbf{1 0}}$ & $2.09 \mathrm{ab} \pm 0.0145$ & $2.05 \mathrm{ab} \pm 0.049$ & $2.07 \mathrm{ab} \pm 0.023$ & $1.95 \mathrm{ab} \pm 0.027$ \\
$\mathbf{T}_{\mathbf{1 1}}$ & $2.13 \mathrm{a} \pm 0.026$ & $2.09 \mathrm{a} \pm 0.034$ & $2.08 \mathrm{a} \pm 0.023$ & $2.02 \mathrm{a} \pm 0.015$ \\
\hline
\end{tabular}

Means within the same column followed by the same letter are not significantly different at $p \leq 0.05$ (Tukey's HSD test). The column represents the mean values \pm standard error of triplicates.

\subsection{Effect of RHB, EFBB, and Dolomitic Limestone treatments on Soil Total Nitrogen}

The repeated measures analysis (Table 3) confirmed that total nitrogen (TN) did not show any significant response to the interaction of treatment combinations and incubation day. In Table 6, it is shown during the 60 days of incubation time that there was no significant effect of biochar and lime application on total nitrogen (TN) among the treatments in acid soil, except for day 15 . The highest $\mathrm{TN}(0.113 \%)$ was obtained in the $\mathrm{T}_{7}$ (75\% lime $\left.+15 \mathrm{t} \mathrm{ha}^{-1} \mathrm{RHB}\right)$ and $\mathrm{T}_{11}\left(75 \%\right.$ lime $\left.+15 \mathrm{t} \mathrm{ha}^{-1} \mathrm{EFBB}\right)$ treatment combination at 15th day and $\mathrm{T}_{9}\left(100 \%\right.$ lime $\left.+10 \mathrm{tha}^{-1} \mathrm{RHB}\right)$ treatment combination at 30th day.

Table 6. Effect of RHB, EFBB, and dolomitic limestone treatments on soil total nitrogen.

\begin{tabular}{ccccc}
\hline \multirow{2}{*}{ Treatment } & \multicolumn{4}{c}{ Total Nitrogen (\%) } \\
\cline { 2 - 5 } & Day 15 & Day 30 & Day 45 & Day 60 \\
\hline $\mathbf{T}_{\mathbf{1}}$ & $0.093 \mathrm{~b} \pm 0.003$ & $0.096 \mathrm{a} \pm 0.013$ & $0.09 \mathrm{a} \pm 0.005$ & $0.100 \mathrm{a} \pm 0.005$ \\
$\mathbf{T}_{\mathbf{2}}$ & $0.106 \mathrm{ab} \pm 0.003$ & $0.100 \mathrm{a} \pm 0.00$ & $0.09 \mathrm{a} \pm 0.00$ & $0.103 \mathrm{a} \pm 0.003$ \\
$\mathbf{T}_{\mathbf{3}}$ & $0.100 \mathrm{ab} \pm 0.00$ & $0.096 \mathrm{a} \pm 0.008$ & $0.08 \mathrm{a} \pm 0.006$ & $0.106 \mathrm{a} \pm 0.003$ \\
$\mathbf{T}_{\mathbf{4}}$ & $0.103 \mathrm{ab} \pm 0.006$ & $0.096 \mathrm{a} \pm 0.003$ & $0.096 \mathrm{a} \pm 0.008$ & $0.100 \mathrm{a} \pm 0.005$ \\
$\mathbf{T}_{\mathbf{5}}$ & $0.100 \mathrm{ab} \pm 0.01$ & $0.100 \mathrm{a} \pm 0.005$ & $0.103 \mathrm{a} \pm 0.008$ & $0.11 \mathrm{a} \pm 0.005$ \\
$\mathbf{T}_{\mathbf{6}}$ & $0.103 \mathrm{ab} \pm 0.006$ & $0.103 \mathrm{a} \pm 0.008$ & $0.11 \mathrm{a} \pm 0.005$ & $0.103 \mathrm{a} \pm 0.003$ \\
$\mathbf{T}_{\mathbf{7}}$ & $0.113 \mathrm{a} \pm 0.006$ & $0.110 \mathrm{a} \pm 0.00$ & $0.11 \mathrm{a} \pm 0.003$ & $0.11 \mathrm{a} \pm 0.005$ \\
$\mathbf{T}_{\mathbf{8}}$ & $0.103 \mathrm{ab} \pm 0.008$ & $0.103 \mathrm{a} \pm 0.006$ & $0.103 \mathrm{a} \pm 0.003$ & $0.103 \mathrm{a} \pm 0.006$ \\
$\mathbf{T}_{\mathbf{9}}$ & $0.103 \mathrm{ab} \pm 0.006$ & $0.113 \mathrm{a} \pm 0.006$ & $0.11 \mathrm{a} \pm 0.005$ & $0.11 \mathrm{a} \pm 0.006$ \\
$\mathbf{T}_{\mathbf{1 0}}$ & $0.103 \mathrm{ab} \pm 0.006$ & $0.103 \mathrm{a} \pm 0.006$ & $0.096 \mathrm{a} \pm 0.003$ & $0.11 \mathrm{a} \pm 0.005$ \\
$\mathbf{T}_{\mathbf{1 1}}$ & $0.113 \mathrm{a} \pm 0.006$ & $0.103 \mathrm{a} \pm 0.006$ & $0.100 \mathrm{a} \pm 0.005$ & $0.096 \mathrm{a} \pm 0.003$ \\
\hline
\end{tabular}

Means within the same column followed by the same letter are not significantly different at $p \leq 0.05$ (Tukey's HSD test). The column represents the mean values \pm standard error of triplicates.

\subsection{Effect of RHB, EFBB, and Dolomitic Limestone Treatments on Soil Available P}

The studied soil had low available P. After applying dolomitic limestone and biochar, soil available $\mathrm{P}$ was significantly increased (Table 7). There were also significant interactions between treatment combinations and incubation days shown in Table 3. The maximum soil available $\mathrm{P}$ of $24.81 \mathrm{mg} \mathrm{kg}^{-1}$ was observed in $\mathrm{T}_{5}\left(100 \%\right.$ lime $\left.+10 \mathrm{tha}^{-1} \mathrm{RHB}\right)$ on the 30th day of incubation, followed by $\mathrm{T}_{7}\left(75 \%\right.$ lime $\left.+15 \mathrm{tha}^{-1} \mathrm{RHB}\right)$; after the 30th day, the availability of $\mathrm{P}$ was decreased. On the 30th day, the highest increment of available $\mathrm{P}$ was $363.74 \%$ from $\mathrm{T}_{5}$ compared to the control. 
Table 7. Effect of RHB, EFBB, and dolomitic limestone treatments on soil available P.

\begin{tabular}{ccccc}
\hline \multirow{2}{*}{ Treatment } & \multicolumn{4}{c}{ Available P $\left.\mathbf{( m g ~ k g} \mathbf{~}^{-\mathbf{1}}\right)$} \\
\cline { 2 - 5 } & Day 15 & Day 30 & Day 45 & Day 60 \\
\hline $\mathbf{T}_{\mathbf{1}}$ & $5.24 \mathrm{e} \pm 0.23$ & $5.35 \mathrm{e} \pm 0.22$ & $5.34 \mathrm{~h} \pm 0.15$ & $5.28 \mathrm{~g} \pm 0.1$ \\
$\mathbf{T}_{\mathbf{2}}$ & $5.48 \mathrm{e} \pm 0.28$ & $6.38 \mathrm{e} \pm 0.25$ & $6.21 \mathrm{~g} \pm 0.20$ & $5.51 \mathrm{~g} \pm 0.19$ \\
$\mathbf{T}_{\mathbf{3}}$ & $6.44 \mathrm{e} \pm 0.25$ & $7.27 \mathrm{e} \pm 0.33$ & $6.90 \mathrm{~g} \pm 0.12$ & $5.98 \mathrm{~g} \pm 0.05$ \\
$\mathbf{T}_{\mathbf{4}}$ & $15.98 \mathrm{~b} \pm 0.62$ & $20.82 \mathrm{~b} \pm 0.55$ & $14.34 \mathrm{~b} \pm 0.19$ & $11.35 \mathrm{bc} \pm 0.17$ \\
$\mathbf{T}_{\mathbf{5}}$ & $18.67 \mathrm{a} \pm 0.75$ & $24.81 \mathrm{a} \pm 0.76$ & $16.43 \mathrm{a} \pm 0.21$ & $13.83 \mathrm{a} \pm 0.21$ \\
$\mathbf{T}_{\mathbf{6}}$ & $13.40 \mathrm{c} \pm 0.76$ & $17.61 \mathrm{c} \pm 0.86$ & $12.08 \mathrm{c} \pm 0.26$ & $10.51 \mathrm{~cd} \pm 0.25$ \\
$\mathbf{T}_{\mathbf{7}}$ & $16.66 \mathrm{ab} \pm 0.73$ & $22.44 \mathrm{ab} \pm 0.81$ & $14.53 \mathrm{~b} \pm 0.26$ & $11.70 \mathrm{~b} \pm 0.25$ \\
$\mathbf{T}_{\mathbf{8}}$ & $10.92 \mathrm{~d} \pm 0.51$ & $15.46 \mathrm{c} \pm 0.70$ & $9.35 \mathrm{e} \pm 0.14$ & $8.22 \mathrm{f} \pm 0.15$ \\
$\mathbf{T}_{\mathbf{9}}$ & $11.76 \mathrm{~cd} \pm 0.64$ & $16.61 \mathrm{c} \pm 0.71$ & $10.68 \mathrm{~d} \pm 0.21$ & $9.77 \mathrm{de} \pm 0.09$ \\
$\mathbf{T}_{\mathbf{1 0}}$ & $9.86 \mathrm{~d} \pm 0.47$ & $11.63 \mathrm{~d} \pm 0.69$ & $8.34 \mathrm{f} \pm 0.18$ & $7.67 \mathrm{f} \pm 0.08$ \\
$\mathbf{T}_{\mathbf{1 1}}$ & $11.63 \mathrm{~cd} \pm 0.70$ & $16.76 \mathrm{c} \pm 1.2$ & $9.65 \mathrm{e} \pm 0.14$ & $9.41 \mathrm{e} \pm 0.16$ \\
\hline
\end{tabular}

Means within the same column followed by the same letter are not significantly different at $p \leq 0.05$ (Tukey's HSD test). The column represents the mean values \pm standard error of triplicates.

\subsection{Effect of RHB, EFBB, and Dolomitic Limestone Treatments on Soil Exchangeable K}

The repeated measures analysis (Table 3) mentioned significant interaction effects of treatment combinations and incubation day on soil exchangeable $\mathrm{K}$. There was also a significant effect of RHB, EFBB, and dolomitic limestone addition on soil exchangeable $\mathrm{K}$ showed in Table 8 . Soil exchangeable $\mathrm{K}$ was highest $\left(1.53 \mathrm{cmol}_{\mathrm{c}} \mathrm{kg}^{-1}\right)$ in soil amended with $75 \%$ lime $+15 \mathrm{tha}^{-1} \mathrm{RHB}\left(\mathrm{T}_{7}\right)$ followed by $\mathrm{T}_{5}\left(1.49 \mathrm{cmol}_{\mathrm{c}} \mathrm{kg}^{-1}\right)$ and $\mathrm{T}_{11}$ $\left(1.48 \mathrm{cmol}_{\mathrm{c}} \mathrm{kg}^{-1}\right)$ on day 60 . The lowest exchangeable $\mathrm{K}$ was found in the control treatment, $\mathrm{T}_{1}\left(0.18 \mathrm{cmol}_{\mathrm{C}} \mathrm{kg}^{-1}\right)$ on day 15 . The exchangeable $\mathrm{K}$ was highest increased by $427.59 \%$ from $\mathrm{T}_{7}\left(75 \%\right.$ lime $\left.+15 \mathrm{tha}^{-1} \mathrm{RHB}\right)$ compared to the control on day 60 .

Table 8. Effect of RHB, EFBB, and dolomitic limestone treatments on soil exchangeable K.

\begin{tabular}{ccccc}
\hline \multirow{2}{*}{ Treatment } & \multicolumn{4}{c}{ Exchangeable $\left.\mathbf{K} \mathbf{~} \mathbf{c m o l}_{\mathbf{c}} \mathbf{~ k g}^{-\mathbf{1}}\right)$} \\
\cline { 2 - 5 } & Day 15 & Day 30 & Day 45 & Day 60 \\
\hline $\mathbf{T}_{\mathbf{1}}$ & $0.18 \mathrm{c} \pm 0.017$ & $0.25 \mathrm{c} \pm 0.023$ & $0.24 \mathrm{~d} \pm 0.023$ & $0.29 \mathrm{~d} \pm 0.015$ \\
$\mathbf{T}_{\mathbf{2}}$ & $0.25 \mathrm{c} \pm 0.015$ & $0.27 \mathrm{c} \pm 0.015$ & $0.29 \mathrm{~d} \pm 0.020$ & $0.33 \mathrm{~d} \pm 0.018$ \\
$\mathbf{T}_{\mathbf{3}}$ & $0.30 \mathrm{c} \pm 0.02$ & $0.32 \mathrm{c} \pm 0.017$ & $0.34 \mathrm{~d} \pm 0.027$ & $0.35 \mathrm{~d} \pm 0.018$ \\
$\mathbf{T}_{\mathbf{4}}$ & $0.89 \mathrm{~b} \pm 0.02$ & $1.22 \mathrm{a} \pm 0.017$ & $1.29 \mathrm{bc} \pm 0.027$ & $1.31 \mathrm{c} \pm 0.017$ \\
$\mathbf{T}_{\mathbf{5}}$ & $0.97 \mathrm{ab} \pm 0.011$ & $1.28 \mathrm{a} \pm 0.022$ & $1.45 \mathrm{a} \pm 0.029$ & $1.49 \mathrm{a} \pm 0.011$ \\
$\mathbf{T}_{\mathbf{6}}$ & $0.90 \mathrm{~b} \pm 0.047$ & $1.04 \mathrm{~b} \pm 0.027$ & $1.28 \mathrm{bc} \pm 0.040$ & $1.30 \mathrm{c} \pm 0.02$ \\
$\mathbf{T}_{\mathbf{7}}$ & $0.99 \mathrm{ab} \pm 0.012$ & $1.35 \mathrm{a} \pm 0.032$ & $1.41 \mathrm{ab} \pm 0.003$ & $1.53 \mathrm{a} \pm 0.017$ \\
$\mathbf{T}_{\mathbf{8}}$ & $0.97 \mathrm{ab} \pm 0.038$ & $1.24 \mathrm{a} \pm 0.037$ & $1.29 \mathrm{bc} \pm 0.013$ & $1.29 \mathrm{c} \pm 0.008$ \\
$\mathbf{T}_{\mathbf{9}}$ & $0.98 \mathrm{ab} \pm 0.029$ & $1.29 \mathrm{a} \pm 0.037$ & $1.31 \mathrm{bc} \pm 0.026$ & $1.40 \mathrm{~b} \pm 0.017$ \\
$\mathbf{T}_{\mathbf{1 0}}$ & $0.93 \mathrm{ab} \pm 0.012$ & $1.03 \mathrm{~b} \pm 0.028$ & $1.22 \mathrm{c} \pm 0.029$ & $1.29 \mathrm{c} \pm 0.017$ \\
$\mathbf{T}_{\mathbf{1 1}}$ & $1.05 \mathrm{a} \pm 0.02$ & $1.29 \mathrm{a} \pm 0.042$ & $1.40 \mathrm{ab} \pm 0.039$ & $1.48 \mathrm{a} \pm 0.024$ \\
\hline
\end{tabular}

Means within the same column followed by the same letter are not significantly different at $p \leq 0.05$ (Tukey's HSD test). The column represents the mean values \pm standard error of triplicates.

\subsection{Effect of RHB, EFBB and Dolomitic Limestone Treatments on Soil Exchangeable Ca}

The exchangeable $\mathrm{Ca}$ of soil under this study was significantly influenced by the amendments and incubation time (Table 3). Data in Table 9 represented the significant effect of biochar and lime amendment on exchangeable Ca. The maximum $\left(4.28 \mathrm{cmol}_{\mathrm{c} \mathrm{kg}} \mathrm{kg}^{-1}\right)$ exchangeable Ca was found in $100 \%$ lime $+10 \mathrm{tha}^{-1} \mathrm{RHB}\left(\mathrm{T}_{5}\right)$ treatment, followed by $100 \%$ lime $+10 \mathrm{tha}^{-1} \mathrm{EFBB}\left(\mathrm{T}_{9}\right)$ on the 60th day of incubation and the minimum exchangeable $\mathrm{Ca}\left(1.33 \mathrm{cmol}_{\mathrm{c}} \mathrm{kg}^{-1}\right)$ found from the unamended soil, $\mathrm{T}_{1}$. The highest exchangeable $\mathrm{Ca}$ was increased by $221.80 \%$ from $\mathrm{T}_{5}\left(100 \%\right.$ lime $\left.+10 \mathrm{t} \mathrm{ha}^{-1} \mathrm{RHB}\right)$ on day 60 compared to the control. 
Table 9. Effect of RHB, EFBB and dolomitic limestone treatments on soil exchangeable Ca.

\begin{tabular}{ccccc}
\hline \multirow{2}{*}{ Treatment } & \multicolumn{4}{c}{ Exchangeable Ca $\mathbf{~ c m o l ~}_{\mathbf{c}} \mathbf{~ k g}^{-\mathbf{1}} \mathbf{~}$} \\
\cline { 2 - 5 } & Day 15 & Day 30 & Day 45 & Day 60 \\
\hline $\mathbf{T}_{\mathbf{1}}$ & $1.39 \mathrm{~h} \pm 0.032$ & $1.33 \mathrm{~h} \pm 0.029$ & $1.37 \mathrm{f} \pm 0.067$ & $1.33 \mathrm{~h} \pm 0.03$ \\
$\mathbf{T}_{\mathbf{2}}$ & $1.40 \mathrm{~h} \pm 0.015$ & $1.36 \mathrm{~h} \pm 0.058$ & $1.40 \mathrm{f} \pm 0.008$ & $1.37 \mathrm{~h} \pm 0.02$ \\
$\mathbf{T}_{\mathbf{3}}$ & $3.19 \mathrm{~d} \pm 0.024$ & $3.14 \mathrm{ef} \pm 0.023$ & $3.15 \mathrm{~d} \pm 0.018$ & $3.13 \mathrm{f} \pm 0.01$ \\
$\mathbf{T}_{\mathbf{4}}$ & $3.11 \mathrm{de} \pm 0.015$ & $3.51 \mathrm{~d} \pm 0.01$ & $3.50 \mathrm{c} \pm 0.029$ & $3.56 \mathrm{~d} \pm 0.005$ \\
$\mathbf{T}_{\mathbf{5}}$ & $3.88 \mathrm{a} \pm 0.024$ & $4.22 \mathrm{a} \pm 0.029$ & $4.20 \mathrm{a} \pm 0.024$ & $4.28 \mathrm{a} \pm 0.01$ \\
$\mathbf{T}_{\mathbf{6}}$ & $2.74 \mathrm{f} \pm 0.017$ & $3.05 \mathrm{f} \pm 0.018$ & $3.06 \mathrm{de} \pm 0.008$ & $3.08 \mathrm{f} \pm 0.10$ \\
$\mathbf{T}_{\mathbf{7}}$ & $2.99 \mathrm{e} \pm 0.014$ & $3.73 \mathrm{c} \pm 0.015$ & $3.88 \mathrm{~b} \pm 0.038$ & $3.91 \mathrm{c} \pm 0.02$ \\
$\mathbf{T}_{\mathbf{8}}$ & $3.33 \mathrm{c} \pm 0.032$ & $3.20 \mathrm{e} \pm 0.031$ & $3.40 \mathrm{c} \pm 0.025$ & $3.44 \mathrm{e} \pm 0.005$ \\
$\mathbf{T}_{\mathbf{9}}$ & $3.78 \mathrm{ab} \pm 0.028$ & $4.01 \mathrm{~b} \pm 0.024$ & $4.05 \mathrm{a} \pm 0.02$ & $4.06 \mathrm{~b} \pm 0.012$ \\
$\mathbf{T}_{\mathbf{1 0}}$ & $2.32 \mathrm{~g} \pm 0.023$ & $2.79 \mathrm{~g} \pm 0.028$ & $2.96 \mathrm{e} \pm 0.015$ & $2.98 \mathrm{~g} \pm 0.01$ \\
$\mathbf{T}_{\mathbf{1 1}}$ & $3.72 \mathrm{~b} \pm 0.028$ & $3.71 \mathrm{c} \pm 0.044$ & $3.82 \mathrm{~b} \pm 0.028$ & $3.88 \mathrm{c} \pm 0.01$ \\
\hline
\end{tabular}

Means within the same column followed by the same letter are not significantly different at $p \leq 0.05$ (Tukey's HSD test). The column represents the mean values \pm standard error of triplicates.

\subsection{Effect of RHB, EFBB, and Dolomitic Limestone Treatments on Soil Exchangeable Mg}

The repeated measures analysis (Table 3) showed that treatment combinations and incubation days significantly affected soil exchangeable Mg. Table 10 shows that organic amendments and lime applications also significantly changed exchangeable $\mathrm{Mg}$ in the soil. The highest exchangeable $\mathrm{Mg}\left(1.53 \mathrm{cmol}_{\mathrm{c}} \mathrm{kg}^{-1}\right)$ was observed in $100 \%$ lime $+10 \mathrm{tha}^{-1}$ EFBB $\left(\mathrm{T}_{9}\right)$ followed by $100 \%$ lime $+10 \mathrm{tha} \mathrm{h}^{-1} \mathrm{RHB}\left(\mathrm{T}_{5}\right)$ on day 60 . The lowest value $\left(0.34 \mathrm{cmol}_{\mathrm{c}} \mathrm{kg}^{-1}\right)$ was noted from control treatment on the 45th day of incubation. The highest increment was $313.51 \%$ from $\mathrm{T}_{9}\left(100 \%\right.$ lime $\left.+10 \mathrm{tha}^{-1} \mathrm{EFBB}\right)$ on day 60 compared to the untreated soil.

Table 10. Effect of RHB, EFBB, and dolomitic limestone treatments on soil exchangeable $\mathrm{Mg}$.

\begin{tabular}{ccccc}
\hline \multirow{2}{*}{ Treatment } & \multicolumn{4}{c}{ Exchangeable $\mathbf{~ M g} \mathbf{~} \mathbf{c m o l}_{\mathbf{c}} \mathbf{~ k g}^{-\mathbf{1}} \mathbf{)}$} \\
\cline { 2 - 5 } & Day $\mathbf{1 5}$ & Day 30 & Day 45 & Day 60 \\
\hline $\mathbf{T}_{\mathbf{1}}$ & $0.39 \mathrm{~h} \pm 0.008$ & $0.37 \mathrm{~d} \pm 0.019$ & $0.34 \mathrm{f} \pm 0.019$ & $0.37 \mathrm{~g} \pm 0.026$ \\
$\mathbf{T}_{\mathbf{2}}$ & $0.40 \mathrm{~h} \pm 0.02$ & $0.39 \mathrm{~d} \pm 0.015$ & $0.37 \mathrm{f} \pm 0.02$ & $0.40 \mathrm{~g} \pm 0.017$ \\
$\mathbf{T}_{\mathbf{3}}$ & $1.39 \mathrm{a} \pm 0.012$ & $1.10 \mathrm{c} \pm 0.021$ & $1.03 \mathrm{e} \pm 0.015$ & $0.97 \mathrm{f} \pm 0.011$ \\
$\mathbf{T}_{\mathbf{4}}$ & $0.96 \mathrm{f} \pm 0.012$ & $1.17 \mathrm{c} \pm 0.021$ & $1.33 \mathrm{~b} \pm 0.025$ & $1.34 \mathrm{~d} \pm 0.02$ \\
$\mathbf{T}_{\mathbf{5}}$ & $1.03 \mathrm{e} \pm 0.012$ & $1.39 \mathrm{a} \pm 0.015$ & $1.43 \mathrm{a} \pm 0.024$ & $1.52 \mathrm{ab} \pm 0.017$ \\
$\mathbf{T}_{\mathbf{6}}$ & $0.89 \mathrm{~g} \pm 0.012$ & $1.11 \mathrm{c} \pm 0.008$ & $1.17 \mathrm{~d} \pm 0.025$ & $1.20 \mathrm{e} \pm 0.014$ \\
$\mathbf{T}_{\mathbf{7}}$ & $1.23 \mathrm{~d} \pm 0.008$ & $1.29 \mathrm{~b} \pm 0.015$ & $1.30 \mathrm{bc} \pm 0.015$ & $1.43 \mathrm{c} \pm 0.008$ \\
$\mathbf{T}_{\mathbf{8}}$ & $1.18 \mathrm{~cd} \pm 0.022$ & $1.32 \mathrm{ab} \pm 0.008$ & $1.31 \mathrm{~b} \pm 0.019$ & $1.34 \mathrm{~d} \pm 0.02$ \\
$\mathbf{T}_{\mathbf{9}}$ & $1.22 \mathrm{c} \pm 0.006$ & $1.29 \mathrm{~b} \pm 0.021$ & $1.40 \mathrm{ab} \pm 0.015$ & $1.53 \mathrm{a} \pm 0.019$ \\
$\mathbf{T}_{\mathbf{1 0}}$ & $0.98 \mathrm{ef} \pm 0.006$ & $1.14 \mathrm{c} \pm 0.015$ & $1.21 \mathrm{~cd} \pm 0.017$ & $1.23 \mathrm{e} \pm 0.008$ \\
$\mathbf{T}_{\mathbf{1 1}}$ & $1.31 \mathrm{~b} \pm 0.008$ & $1.36 \mathrm{ab} \pm 0.021$ & $1.37 \mathrm{ab} \pm 0.018$ & $1.45 \mathrm{bc} \pm 0.014$ \\
\hline
\end{tabular}

Means within the same column followed by the same letter are not significantly different at $p \leq 0.05$ (Tukey's HSD test). The column represents the mean values \pm standard error of triplicates.

\subsection{Effect of RHB, EFBB, and Dolomitic Limestone Treatments on Soil Exchangeable Al}

The significant effect of dolomitic limestone and different types of biochar on exchangeable $\mathrm{Al}$ is presented in Table 11. In addition, the significant interaction effects of treatments and incubation days are presented in Table 3. The exchangeable Al significantly decreased due to lime and biochar treatments. Initially, the value decreased drastically up to 15 days of incubation, and thereafter it continued slowly (Table 11). The lowest value $\left(0.00 \mathrm{cmol}_{\mathrm{c}} \mathrm{kg}^{-1}\right)$ was noted from $\mathrm{T}_{5}\left(100 \%\right.$ lime $\left.+10 \mathrm{t} \mathrm{ha}^{-1} \mathrm{RHB}\right), \mathrm{T}_{7}$ (75\% lime $\left.+15 \mathrm{t} \mathrm{ha}^{-1} \mathrm{RHB}\right)$, and $\mathrm{T}_{11}\left(75 \%\right.$ lime $\left.+15.0 \mathrm{tha}^{-1} \mathrm{EFBB}\right)$ and the highest value $\left(2.38 \mathrm{cmol}_{\mathrm{c}} \mathrm{kg}^{-1}\right)$ found from control on 60th day. 
Table 11. Effect of RHB, EFBB, and dolomitic limestone treatments on soil exchangeable Al.

\begin{tabular}{|c|c|c|c|c|}
\hline \multirow{2}{*}{ Treatment } & \multicolumn{4}{|c|}{ Exchangeable Al $\left(\mathrm{cmol}_{\mathrm{c}} \mathbf{k g}^{-1}\right)$} \\
\hline & Day 15 & Day 30 & Day 45 & Day 60 \\
\hline $\mathbf{T}_{1}$ & $2.41 \mathrm{a} \pm 0.01$ & $2.42 \mathrm{a} \pm 0.01$ & $2.40 \mathrm{a} \pm 0.01$ & $2.38 \mathrm{a} \pm 0.01$ \\
\hline $\mathrm{T}_{2}$ & $2.05 b \pm 0.02$ & $1.69 b \pm 0.02$ & $1.57 \mathrm{~b} \pm 0.01$ & $1.21 b \pm 0.01$ \\
\hline $\mathrm{T}_{3}$ & $0.79 c \pm 0.01$ & $0.64 c \pm 0.01$ & $0.35 c \pm 0.02$ & $0.03 \mathrm{~d} \pm 0.01$ \\
\hline $\mathrm{T}_{4}$ & $0.06 \mathrm{e} \pm 0.01$ & $0.04 \mathrm{e} \pm 0.01$ & $0.04 \mathrm{e} \pm 0.01$ & $0.01 \mathrm{~d} \pm 0.00$ \\
\hline $\mathrm{T}_{5}$ & $0.03 e \pm 0.02$ & $0.02 \mathrm{e} \pm 0.01$ & $0.00 \mathrm{e} \pm 0.00$ & $0.00 \mathrm{~d} \pm 0.00$ \\
\hline $\mathrm{T}_{6}$ & $0.39 \mathrm{~d} \pm 0.01$ & $0.32 \mathrm{~d} \pm 0.01$ & $0.29 \mathrm{~d} \pm 0.01$ & $0.13 c \pm 0.02$ \\
\hline $\mathrm{T}_{7}$ & $0.03 \mathrm{e} \pm 0.01$ & $0.02 \mathrm{e} \pm 0.01$ & $0.01 \mathrm{e} \pm 0.00$ & $0.00 \mathrm{~d} \pm 0.00$ \\
\hline $\mathrm{T}_{8}$ & $0.07 \mathrm{e} \pm 0.01$ & $0.05 \mathrm{e} \pm 0.01$ & $0.02 \mathrm{e} \pm 0.01$ & $0.02 \mathrm{~d} \pm 0.01$ \\
\hline $\mathrm{T}_{9}$ & $0.06 \mathrm{e} \pm 0.01$ & $0.03 e \pm 0.01$ & $0.01 \mathrm{e} \pm 0.00$ & $0.02 \mathrm{~d} \pm 0.00$ \\
\hline $\mathbf{T}_{10}$ & $0.42 \mathrm{~d} \pm 0.01$ & $0.33 d \pm 0.01$ & $0.28 \mathrm{~d} \pm 0.01$ & $0.17 \mathrm{c} \pm 0.02$ \\
\hline$T_{11}$ & $0.03 \mathrm{e} \pm 0.01$ & $0.03 \mathrm{e} \pm 0.01$ & $0.02 \mathrm{e} \pm 0.01$ & $0.00 \mathrm{~d} \pm 0.00$ \\
\hline
\end{tabular}

Means within the same column followed by the same letter are not significantly different at $p \leq 0.05$ (Tukey's HSD test). The column represents the mean values \pm standard error of triplicates.

\subsection{Effect of RHB, EFBB, and Dolomitic Limestone Treatments on Soil Extractable Fe}

The significant influence of treatment combinations with RHB, EFBB, and dolomitic limestone on extractable Fe is shown in Table 12. The repeated measures analysis showed that treatment combinations and incubation days significantly affected soil extractable Fe in Table 3. The value of extractable Fe was significantly different with values ranging from 41.48 to $90.89 \mathrm{mg} \mathrm{kg}^{-1}$. The lowest value $\left(41.48 \mathrm{mg} \mathrm{kg}^{-1}\right)$ of Fe was found from $\mathrm{T}_{7}$ (75\% lime $\left.+15 \mathrm{tha}^{-1} \mathrm{RHB}\right)$, and this value decreased by $50.99 \%$ on the 30 th day of incubation. The highest value $\left(90.89 \mathrm{mg} \mathrm{kg}^{-1}\right)$ was revealed by the control $\left(\mathrm{T}_{1}\right)$ treatment on the 15th day of incubation.

Table 12. Effect of RHB, EFBB, and dolomitic limestone treatments on soil extractable Fe.

\begin{tabular}{|c|c|c|c|c|}
\hline \multirow{2}{*}{ Treatment } & \multicolumn{4}{|c|}{ Extractable Fe (mg kg $\left.{ }^{-1}\right)$} \\
\hline & Day 15 & Day 30 & Day 45 & Day 60 \\
\hline $\mathbf{T}_{1}$ & $90.89 \mathrm{a} \pm 2.01$ & $84.63 a \pm 0.38$ & $82.41 \mathrm{a} \pm 1.74$ & $83.08 \mathrm{a} \pm 0.96$ \\
\hline $\mathbf{T}_{2}$ & $78.23 b \pm 1.82$ & $75.64 b \pm 0.34$ & $77.41 b \pm 0.78$ & $78.75 b \pm 1.11$ \\
\hline$T_{3}$ & $54.72 c \pm 1.59$ & $67.45 c \pm 0.88$ & $71.08 \mathrm{c} \pm 0.66$ & $71.75 c \pm 1.11$ \\
\hline $\mathrm{T}_{4}$ & $47.97 \mathrm{~cd} \pm 0.99$ & $53.33 \mathrm{ef} \pm 0.45$ & $59.02 d \pm 0.62$ & $61.34 \mathrm{e} \pm 0.84$ \\
\hline$T_{5}$ & $44.83 \mathrm{~d} \pm 1.59$ & $42.33 h \pm 0.86$ & $42.56 f \pm 0.79$ & $48.90 \mathrm{~g} \pm 0.90$ \\
\hline$T_{6}$ & $54.48 \mathrm{c} \pm 1.33$ & $61.55 \mathrm{~d} \pm 0.72$ & $67.13 c \pm 1.22$ & $66.63 \mathrm{~d} \pm 0.96$ \\
\hline $\mathbf{T}_{7}$ & $44.13 \mathrm{~d} \pm 1.97$ & $41.48 \mathrm{~h} \pm 0.33$ & $43.36 f \pm 0.68$ & $47.70 \mathrm{~g} \pm 0.92$ \\
\hline $\mathrm{T}_{8}$ & $49.02 \mathrm{~cd} \pm 2.02$ & $54.68 \mathrm{e} \pm 0.59$ & $54.71 \mathrm{ed} \pm 0.62$ & $55.71 \mathrm{f} \pm 0.62$ \\
\hline $\mathrm{T}_{9}$ & $46.01 \mathrm{~d} \pm 0.83$ & $49.68 \mathrm{fg} \pm 1.52$ & $53.36 \mathrm{e} \pm 0.74$ & $54.70 \mathrm{f} \pm 0.79$ \\
\hline $\mathbf{T}_{10}$ & $55.75 c \pm 1.21$ & $61.69 \mathrm{~d} \pm 0.82$ & $68.85 c \pm 0.81$ & $67.52 \mathrm{~d} \pm 0.56$ \\
\hline $\mathrm{T}_{11}$ & $46.07 \mathrm{~d} \pm 1.03$ & $48.73 \mathrm{~g} \pm 1.37$ & $55.67 \mathrm{de} \pm 1.52$ & $56.00 \mathrm{f} \pm 0.93$ \\
\hline
\end{tabular}

Means within the same column followed by the same letter are not significantly different at $p \leq 0.05$ (Tukey's HSD test). The column represents the mean values \pm standard error of triplicates. 


\subsection{Effect of RHB, EFBB, and Dolomitic Limestone Treatments on Soil Extractable Mn}

The extractable Mn of soil under this study was significantly influenced by the amendments and incubation time (Table 3). Applying biochar and lime significantly altered the soil extractable Mn, which is shown in Table 13. Application of $75 \%$ lime $+15 \mathrm{t} \mathrm{ha}^{-1} \mathrm{RHB}$ $\left(\mathrm{T}_{7}\right)$ maximized the soil extractable $\mathrm{Mn}\left(7.13 \mathrm{mg} \mathrm{kg}^{-1}\right)$ in soil on day 15 of the incubation period, which was statistically similar to $\mathrm{T}_{5}$ and $\mathrm{T}_{4}$ while the minimum soil extractable $\mathrm{Mn}$ showed by control. The highest increment was $65.43 \%$ from $\mathrm{T}_{7}\left(75 \%\right.$ lime $\left.+15 \mathrm{tha}^{-1} \mathrm{RHB}\right)$ compared to the control.

Table 13. Effect of RHB, EFBB, and dolomitic limestone treatments on soil extractable Mn.

\begin{tabular}{ccccc}
\hline \multirow{2}{*}{ Treatment } & \multicolumn{4}{c}{ Extractable Mn $\left.\mathbf{( m g ~ k g} \mathbf{~}^{-\mathbf{1}}\right)$} \\
\cline { 2 - 5 } & Day $\mathbf{1 5}$ & Day 30 & Day $\mathbf{4 5}$ & Day $\mathbf{6 0}$ \\
\hline $\mathbf{T}_{\mathbf{1}}$ & $4.44 \mathrm{f} \pm 0.01$ & $4.39 \mathrm{j} \pm 0.01$ & $4.58 \mathrm{~h} \pm 0.01$ & $4.41 \mathrm{~h} \pm 0.01$ \\
$\mathbf{T}_{\mathbf{2}}$ & $4.31 \mathrm{~g} \pm 0.02$ & $4.48 \mathrm{i} \pm 0.01$ & $4.68 \mathrm{~g} \pm 0.01$ & $4.58 \mathrm{~g} \pm 0.01$ \\
$\mathbf{T}_{\mathbf{3}}$ & $5.13 \mathrm{e} \pm 0.01$ & $5.16 \mathrm{~h} \pm 0.01$ & $5.11 \mathrm{e} \pm 0.01$ & $4.92 \mathrm{f} \pm 0.01$ \\
$\mathbf{T}_{\mathbf{4}}$ & $7.07 \mathrm{ab} \pm 0.01$ & $5.55 \mathrm{~d} \pm 0.01$ & $5.19 \mathrm{~d} \pm 0.01$ & $5.17 \mathrm{~d} \pm 0.01$ \\
$\mathbf{T}_{\mathbf{5}}$ & $7.12 \mathrm{a} \pm 0.01$ & $5.62 \mathrm{ab} \pm 0.01$ & $5.39 \mathrm{~b} \pm 0.01$ & $5.37 \mathrm{~b} \pm 0.01$ \\
$\mathbf{T}_{\mathbf{6}}$ & $7.03 \mathrm{~b} \pm 0.01$ & $5.29 \mathrm{~g} \pm 0.01$ & $5.31 \mathrm{c} \pm 0.01$ & $5.33 \mathrm{bc} \pm 0.02$ \\
$\mathbf{T}_{\mathbf{7}}$ & $7.13 \mathrm{a} \pm 0.02$ & $5.66 \mathrm{a} \pm 0.02$ & $5.60 \mathrm{a} \pm 0.01$ & $5.48 \mathrm{a} \pm 0.02$ \\
$\mathbf{T}_{\mathbf{8}}$ & $6.11 \mathrm{~d} \pm 0.02$ & $5.49 \mathrm{e} \pm 0.01$ & $5.15 \mathrm{~d} \pm 0.01$ & $5.13 \mathrm{~d} \pm 0.01$ \\
$\mathbf{T}_{\mathbf{9}}$ & $6.26 \mathrm{c} \pm 0.01$ & $5.56 \mathrm{~cd} \pm 0.01$ & $5.17 \mathrm{~d} \pm 0.01$ & $5.16 \mathrm{~d} \pm 0.01$ \\
$\mathbf{T}_{\mathbf{1 0}}$ & $6.04 \mathrm{~d} \pm 0.02$ & $5.39 \mathrm{f} \pm 0.02$ & $5.06 \mathrm{f} \pm 0.01$ & $5.02 \mathrm{e} \pm 0.01$ \\
$\mathbf{T}_{\mathbf{1 1}}$ & $6.21 \mathrm{c} \pm 0.03$ & $5.60 \mathrm{bc} \pm 0.01$ & $5.34 \mathrm{c} \pm 0.01$ & $5.27 \mathrm{c} \pm 0.01$ \\
\hline
\end{tabular}

Means within the same column followed by the same letter are not significantly different at $p \leq 0.05$ (Tukey's HSD test). The column represents the mean values \pm standard error of triplicates.

\subsection{Effect of RHB, EFBB, and Dolomitic Limestone Treatments on Soil $\mathrm{CO}_{2}$ Emission}

The effect of biochar and lime application on $\mathrm{CO}_{2}$ emission from the Ultisol under study is shown in Figure 2, while the cumulative $\mathrm{CO}_{2}$ emissions are depicted in Figure 3. The repeated measures analysis (Table 3 ) on the soil $\mathrm{CO}_{2}$ emission illustrated significant interaction effects of treatment combinations and incubation day. There was higher $\mathrm{CO}_{2}$ efflux observed in the oil palm EFB biochar treatment compared to that emitted by RHB. The highest $\mathrm{CO}_{2}$ efflux resulted within the first $24 \mathrm{~h}$ of incubation, and the magnitude of the increment was more than four times greater for all the biochar treated soil. There was a sharp decline on day 2 after the peak emission of $\mathrm{CO}_{2}$ efflux on day 1 , and a gradual decrease occurs during the whole incubation period. Afterwards, from all treatments, the $\mathrm{CO}_{2}$ emission remained steady with little emission. Among the treatments, the highest cumulative $\mathrm{CO}_{2}$ emission appeared $272.69 \mu \mathrm{mol} \mathrm{CO} \mathrm{m}^{-2}$ from $\mathrm{T}_{9},\left(100 \%\right.$ lime $\left.+15 \mathrm{t} \mathrm{ha}^{-1} \mathrm{EFBB}\right)$ followed by $\mathrm{T}_{11}\left(266.87 \mu \mathrm{mol} \mathrm{CO} \mathrm{m}^{-2}\right)$ and $\mathrm{T}_{8}\left(260.20 \mu \mathrm{mol} \mathrm{CO}_{2} \mathrm{~m}^{-2}\right)$. There were significant differences in $\mathrm{CO}_{2}$ emission between the treatments $\mathrm{T}_{9}$ and $\mathrm{T}_{11}$. The lowest cumulative $\mathrm{CO}_{2}$ efflux of $86.63 \mu \mathrm{mol} \mathrm{CO} \mathrm{CO}^{-2}$ occurred in the control treatment. The cumulative $\mathrm{CO}_{2}$ emission was in the order of $\mathrm{T}_{9}>\mathrm{T}_{11}>\mathrm{T}_{8}>\mathrm{T}_{10}>\mathrm{T}_{3}>\mathrm{T}_{5}>\mathrm{T}_{7}>\mathrm{T}_{4}>$ $\mathrm{T}_{6}>\mathrm{T}_{2}>\mathrm{T}_{1}$. Overall, it can be said that the more dolomitic limestone applied, the higher was the $\mathrm{CO}_{2}$ gas emitted. Note that the biochar produced from rice husk released a lower amount of $\mathrm{CO}_{2}$ than that of the biochar from oil palm empty fruit bunches. 


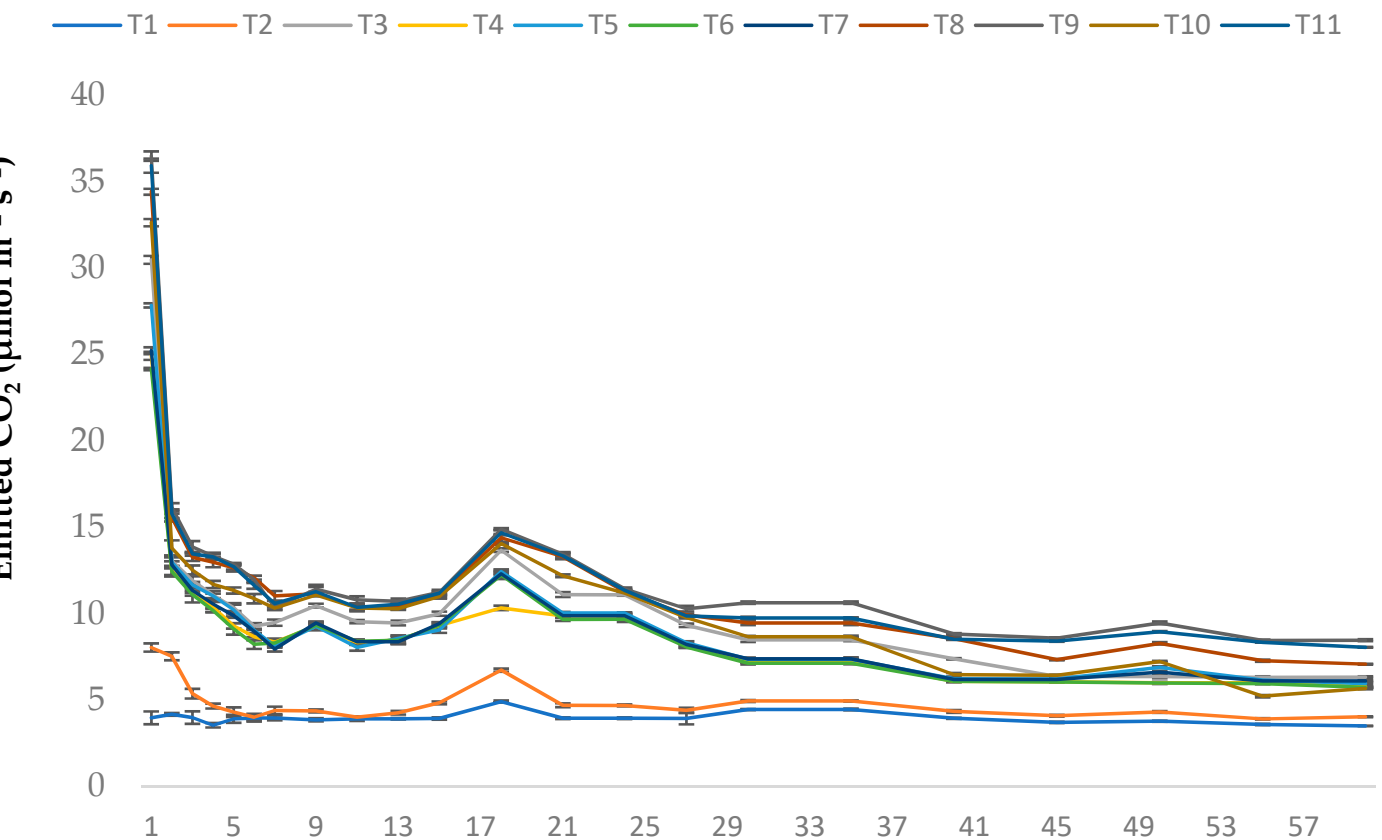

Figure 2. Effect of RHB, EFBB, and dolomitic limestone treatments on soil $\mathrm{CO}_{2}$ emission during the 60 days of incubation. Bar errors show \pm standard error of three replications.

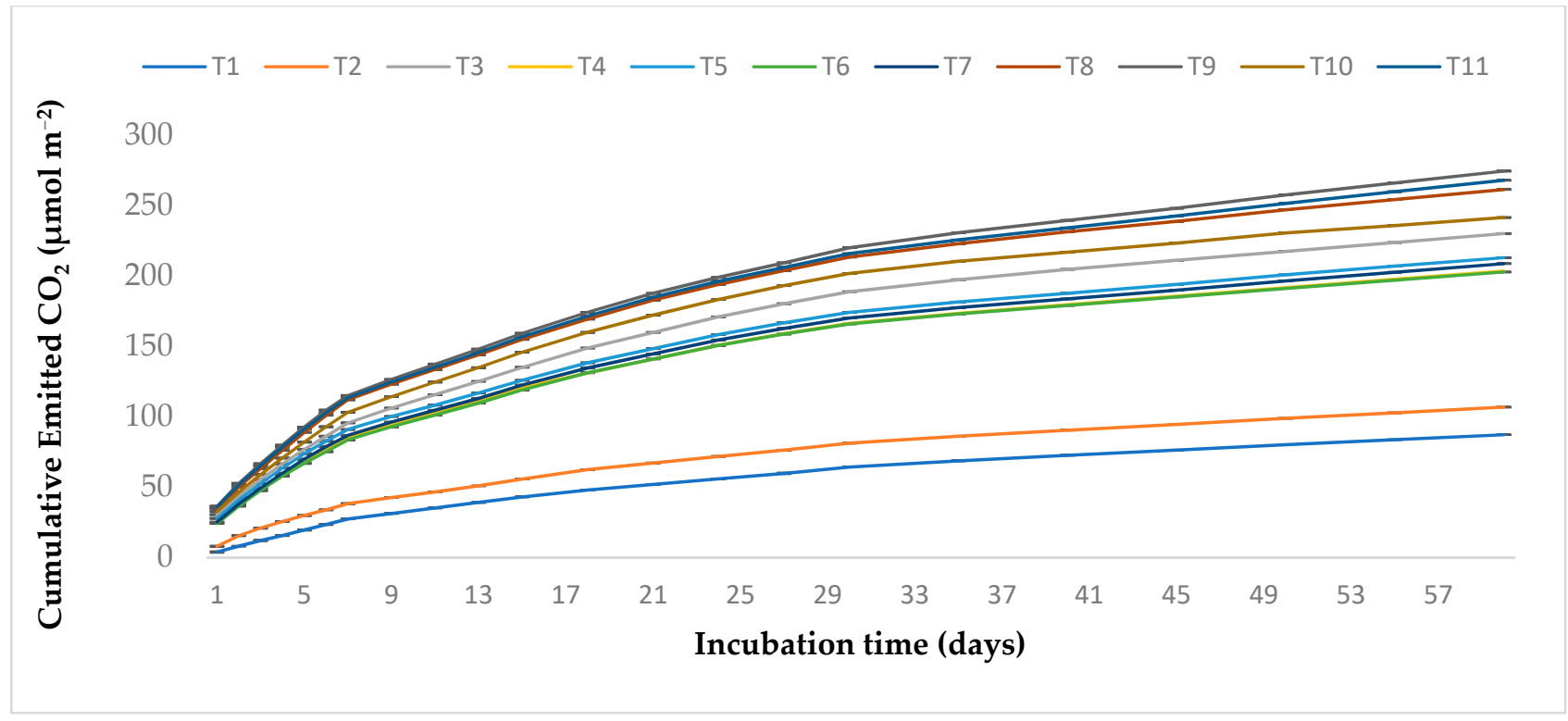

Figure 3. Effect of RHB, EFBB, and dolomitic limestone treatments on cumulative soil $\mathrm{CO}_{2}$ emission during the 60 days of incubation, whereas bar errors show \pm standard error of three replications.

\section{Discussion}

\subsection{Effect of the Amendments on Soil Nutrients}

Previously, many researchers had shown that the organic amendment has the ability to upsurge the soil $\mathrm{pH}[46,47]$. Our study also showed that the soil $\mathrm{pH}$ increased significantly by adding biochar and lime compared to control. The ash content, produced during the pyrolysis process, created an alkaline condition in the rice husk and oil palm empty fruit bunches biochars. Plant ash contains a high amount of basic cations, particularly $\mathrm{Ca}$, which make up the deficiency of $\mathrm{Ca}$ in soil and thus increase soil $\mathrm{pH}[26,48]$. The application of biochar demonstrated a long-lasting effect on soil $\mathrm{pH}$ compared to lime. The increase of soil $\mathrm{pH}$ could also be attributed to the increase of base saturation by the three cations $\left(\mathrm{Ca}^{2+}, \mathrm{K}^{+}\right.$, 
and $\mathrm{Mg}^{2+}$ ) as a result of decreasing the concentration of exchangeable $\mathrm{H}^{+}$and exchangeable $\mathrm{Al}^{3+}$ [49]. Rabileh and co-authors reported an increment in soil $\mathrm{pH}$ by 1.88 units applying $10 \mathrm{t} \mathrm{ha} \mathrm{a}^{-1}$ oil palm empty fruit bunches biochar and $2 \mathrm{t} \mathrm{ha}^{-1}$ dolomitic limestone [26]. They explained that the formation of alkaline oxides or carbonates during the pyrolysis process of biochar could decrease the exchangeable acidity of the soil. The reason for the liming effect of biochar is the high proton consumption capacity of biochars as a result of hydrolysis of high concentrations of positively charged cations $\left(\mathrm{K}^{+}, \mathrm{Ca}^{2+}, \mathrm{Mg}^{2+}\right)[50-52]$.

Table 1 showed that RHB and EFB biochar contained $24.86 \%$ and $52.11 \%$ total carbon, respectively. According to Lehmann et al. [53], high intrinsic carbon content is contained by biochar itself, which causes higher TOC in biochar treated soil. Zhang and colleagues found that the application of $20 \mathrm{tha}^{-1}$ biochar in combination with urea can increase soil organic carbon content by $25 \%$, relative to the unmodified soil [54]. This result is consistent with Sukartono et al. [55], who treated soil with $15 \mathrm{t} \mathrm{ha}^{-1}$ biochar, and the organic carbon content was increased by $27 \%$. Biochar application in the soil increases the content of TOC than the untreated soil because it influences $C$ store and $C$ sequestration to soil due to its recalcitrant characteristic over time [56]. According to Zhang et al. [57], total nitrogen content increases by biochar application as well to soil though the effect is insignificant on soil mineral nitrogen content. Nitrogen in biochar is not readily available for plant uptake due to its chemistry, the aromatic structure [58,59]. During the pyrolysis process, the heterocyclic compounds in biochar, such as amino acid, an amino sugar, and amines, lose most of the nitrogen. This causes nitrogen immobilization, making it unavailable for plants to uptake, and causes the rise in the $\mathrm{C} / \mathrm{N}$ ratio $[60,61]$. Our study is also similar to this relationship.

After applying the soil amendment, the availability of soil P increased up to the first 30 days of incubation; later on, it decreased. This pattern is congruent with the findings of Haque et al. [46]. Mineralization of microbial activities from the soil organic amendments may be the reason for this increment [62,63]. Afterward, available soil $\mathrm{P}$ was fixed by the hydrous oxides of $\mathrm{Al}$ and $\mathrm{Fe}$, which was responsible for the reduction of available $\mathrm{P}$ [64]. This study result is well supported by the results reported by Panhwar and co-authors [65], where they stated a $111.83 \%$ increase of available P by combined application of lime and bio-fertilizer. During the pyrolysis process of organic materials, macronutrients like $\mathrm{P}$ remain in the same amount; thus, in biochar, the concentration of $P$ increases [66]. The availability of $\mathrm{P}$ increases due to the increase in soil $\mathrm{pH}$, exchangeable cation, and reduction of the activities of $\mathrm{Al}$ and $\mathrm{Fe}$ [67]. The conducted research also confirmed such relationships.

In this study, the highest exchangeable $\mathrm{K}\left(1.53 \mathrm{cmol}_{\mathrm{C}} \mathrm{kg}^{-1}\right)$ was found from $\mathrm{T}_{7}$ ( $75 \%$ lime $\left.+15 \mathrm{t} \mathrm{ha}^{-1} \mathrm{RHB}\right)$, and it increased by $427.59 \%$ compared to the control; whereas, $\mathrm{T}_{3}\left(100 \%\right.$ lime) showed the value of exchangeable $\mathrm{K}$ was $0.35 \mathrm{cmol}_{\mathrm{c}} \mathrm{kg}^{-1}$ that increased only $20.69 \%$ compared to the control. According to Zaidun et al., soil exchangeable K was increased by $64.30 \%$ and $111.57 \%$, respectively, by applying $10 \mathrm{tha}^{-1}$ and $20 \mathrm{t} \mathrm{ha}^{-1}$ of EFB-POME(Empty fruit bunch-palm oil mill effluent) biochar [68]. Gautam and colleagues reported a similar finding in silty loam Nepalese soil, where exchangeable $\mathrm{K}$ was increased using biochar at $5 \mathrm{tha}^{-1}$ because of the high content of ash in biochar [69]. The main mechanism of increased soil exchangeable $\mathrm{K}$ with incubation time was the high amount of ash contained by the biochar, which promotes the instant discharge of mineral nutrients to the soil [70], the reduction of K loss through leaching [54,71]. Further, using biochar will increase exchangeable basic cations like $\mathrm{Ca}, \mathrm{Mg}, \mathrm{K}$, and $\mathrm{Na}$, encourage the proliferation of $\mathrm{K}$ solubilizing bacteria, and discharge $\mathrm{K}$ from clay minerals [72]. In our study, the soil exchangeable Ca increased after the application of soil amendments. This result is well supported by the study of Haque et al. [46]. This is also consistent with the study done by Panhwar et al., where lime and RHB were used with or without bio-fertilizer [65]. The exchangeable $\mathrm{Ca}$ content increased due to incorporating calcium-rich organic amendments into the soil [62]. It could be attributed to Table 1 that EFB biochar contained a higher amount of exchangeable Mg than RHB. From Table 9, $\mathrm{T}_{3}$ (100\% lime) showed 162.16\% increased soil exchangeable $\mathrm{Mg}$, but the highest exchangeable $\mathrm{Mg}$ increased by $313.51 \%$ 
from $\mathrm{T}_{9}\left(100 \%\right.$ lime $\left.+10 \mathrm{t} \mathrm{ha}^{-1} \mathrm{EFBB}\right)$ compared to the control. The current study results are in line with those of Panhwar et al. [65], who reported an exchangeable Mg increase by $282.81 \%$ by applying lime and bio-fertilizer. Since several basic cations are present in biochar ash, the content of exchangeable bases can increment with the application of biochar to the soil [73].

A significant negative relationship was found between soil $\mathrm{pH}$ and exchangeable $\mathrm{Al}$ by the studies conducted in the past $[25,68,74]$. Our results demonstrated the same findings. Biochar plays several roles in the $\mathrm{Al}$ toxicity amelioration of the Ultisol under experimental conditions. Besides being alkaline in nature, it contains a significant quantity of basic cations that produce $\mathrm{OH}^{-}$upon their hydrolysis. Furthermore, the biochar surface can act as an absorbent for the carboxyl and phenolic group of $\mathrm{Al}[75,76]$. Applying chicken litter biochar on soil results in an increase of soil $\mathrm{pH}$ by 0.99 units and reduces exchangeable $\mathrm{Al}$ and soluble Fe in the amended soil [26]. A similar result was reported by Nigussie and co-authors [70], who applied $10 \mathrm{tha}^{-1}$ maize stalk biochar on soil that increased soil $\mathrm{pH}$ by 0.49 units. This was attributed to the higher surface area and porous characteristics of the biochar, which decrease with soluble $\mathrm{Al}$ and $\mathrm{Fe}$ in the amended soil. Our result of decreased extractable Fe by $50.99 \%$ is consistent with that of Ch'ng et al. [26], who reported a decreased extractable Fe by $44.49 \%$ due to chicken litter biochar application. A decrease in the dissolvable Fe content with the addition of biochar may be because of its immobilization via chelation by the organic acids released by the biochar $[26,77]$. The significant increment of the extractable Mn might be due to the inherent presence of $\mathrm{Mn}$ in the biochar [78]. This result is in line with that of the study conducted by Devika and co-workers [79], where soil Mn content was increased by $43.51 \%$ with $5 \mathrm{t} \mathrm{ha}^{-1}$ biochar in clay loam soil in India. According to Ullah et al., Mn concentration can be increased by $113.64 \%$ with the addition of $10 \mathrm{t} \mathrm{ha}^{-1}$ wheat straw biochar in sandy loam soil [80]. We also know that biochar can hold a high amount of $\mathrm{Mn}$ since it is associated with an organic and inorganic form of plant-based materials [81].

\subsection{Carbon Dioxide Emission from the Amended Soil}

In our experiment, the highest cumulative $\mathrm{CO}_{2}$ emission was found in $\mathrm{T}_{9}(100 \%$ lime $+10 \mathrm{tha}^{-1} \mathrm{EFBB}$ ), followed by $\mathrm{T}_{11}, \mathrm{~T}_{8}$, and $\mathrm{T}_{10}$. This result seems to indicate that soil amended with oil palm EFB biochar had a greater cumulative $\mathrm{CO}_{2}$ efflux than $\mathrm{RHB}$ amended soil due to the higher carbon content in EFB biochar than RHB [82]. Moreover, the application of biochar and dolomite highly influenced the rate of $\mathrm{CO}_{2}$ emission. The more lime application, the higher the $\mathrm{CO}_{2}$ emission would be due to increased microbial respiration and subsequent production of $\mathrm{CO}_{2}$ [83].

The initial phase of the incubation period produced the highest amount of $\mathrm{CO}_{2}$ emission from all the amended soil. This result is in agreement with that of the studies conducted by others [84-88]. The $\mathrm{CO}_{2}$ emission was the highest $\left(36.49 \mu \mathrm{mol} \mathrm{CO}_{2} \mathrm{~m}^{-2} \mathrm{~s}^{-1}\right)$ on day 1 because of the speedy stimulation of microbial activities [89] and carbonate dissociation [90]. Fast mineralization of the decomposable soil organic carbon (SOC) was responsible for this pattern of $\mathrm{CO}_{2}$ emission [91]. This pattern of $\mathrm{CO}_{2}$ emission is consistent with the result obtained by other researchers [92-95]. Our result showed that a high rate of biochar and lime emitted higher $\mathrm{CO}_{2}$ flux because of higher $\mathrm{CO}_{2}$, which was released due to the rapid microbial activities, which stimulated the prompt degradation of soil organic matter [96].

It is to be noted that the emission of $\mathrm{CO}_{2}$ can be reduced by using biochar due to the labile carbon fraction sorption on the surface of the biochar or into the pore space of biochar $[97,98]$. Another reason for the reduction of $\mathrm{CO}_{2}$ emission was the slow decomposition of biochar with the labile $C$ depleted, and the $C$ structure changed, which contributed to the suppression of microbial activity [99]. On the other hand, biochar applied to the soil can increase the emission of $\mathrm{CO}_{2}$ [100], which is due to the biochar's volatile organic C [101]. 


\section{Conclusions}

The co-application of biochar and lime showed a promising effect on nutrient availability and on reducing $\mathrm{CO}_{2}$ emission by increasing soil $\mathrm{pH}$ in acidic soils. At optimum $\mathrm{pH}$ (6.5 to 7.5), organic carbon mineralization increased, causing higher $\mathrm{CO}_{2}$ emission and reducing the $\mathrm{Al}$ and Fe toxicity. Therefore, applying these amendments to agricultural land can be a potential agronomic practice to enhance soil fertility and crop productivity. The current study results showed that rice husk biochar with $75 \%$ lime had a better performance than the oil palm EFB biochar based on nutrient availability and $\mathrm{CO}_{2}$ emission. We found that biochar with lime treatment increased soil $\mathrm{pH}$, available $\mathrm{P}$, and decreased exchangeable Al. The emission of $\mathrm{CO}_{2}$ was lower in the rice husk biochar treated soil than oil palm EFB biochar treated soil, which dictates that the effect of biochar and lime on soil fertility and crop productivity may vary with biochar properties and different pyrolysis temperatures. Although this study's outcome is very encouraging, extensive field trials are warranted so that farmers can benefit from this research.

Author Contributions: Conceptualization, M.K.U., and M.F.S.; designed experiments, collected data, conducted statistical analysis, M.M., M.K.U., M.F.S. and A.N.A.H.; validation, M.K.U., S.J. and M.F.S.; writing—original draft preparation, M.M., writing—review and editing, M.K.U., S.J., M.F.S. and S.M.S.; visualization, M.M., M.K.U., M.F.S. and A.N.A.H.; supervision, M.K.U. and M.F.S. All authors have read and agreed to the published version of the manuscript.

Funding: This paper was supported by Universiti Putra Malaysia (Vote No. 6282512-10201), Fundamental Research Grant Scheme (FRGS/1/2020/WAB04/UPM/02/16) and National Agricultural Technology Programme (NATP): Phase-II Project, Bangladesh Agricultural Research Council.

Institutional Review Board Statement: Not applicable.

Informed Consent Statement: Not applicable.

Data Availability Statement: Not applicable.

Acknowledgments: Authors are grateful to the National Agricultural Technology Program (NATP): Phase-II Project, Bangladesh Agricultural Research Council for the financial support, and the Universiti Putra Malaysia, Selangor, Darul Ehsan, Malaysia, for the research facilities.

Conflicts of Interest: The authors declare no conflict of interest.

\section{References}

1. Shaaban, M.; Wu, Y.; Peng, Q.-A.; Lin, S.; Mo, Y.; Wu, L.; Hu, R.; Zhou, W. Effects of dicyandiamide and dolomite application on $\mathrm{N} 2 \mathrm{O}$ emission from an acidic soil. Environ. Sci. Pollut. Res. 2015, 23, 6334-6342. [CrossRef]

2. Dai, Z.; Zhang, X.; Tang, C.; Muhammad, N.; Wu, J.; Brookes, P.C.; Xu, J. Potential role of biochars in decreasing soil acidifi-cation-a critical review. Sci. Total Environ. 2017, 581-582, 601-611. [CrossRef]

3. Holland, J.; Bennett, A.; Newton, A.; White, P.; McKenzie, B.; George, T.; Pakeman, R.; Bailey, J.; Fornara, D.; Hayes, R.; et al. Liming impacts on soils, crops and biodiversity in the UK: A review. Sci. Total. Environ. 2018, 610-611, 316-332. [CrossRef]

4. Anda, M.; Shamshuddin, J.; Fauziah, C.; Omar, S.S. Mineralogy and factors controlling charge development of three Oxisols developed from different parent materials. Geoderma 2008, 143, 153-167. [CrossRef]

5. Ryan, P.R. Assessing the role of genetics for improving the yield of Australia's major grain crops on acid soils. Crop Pasture Sci. 2018, 69, 242-264. [CrossRef]

6. Kalkhoran, S.S.; Pannell, D.J.; Thamo, T.; White, B.; Polyakov, M. Soil acidity, lime application, nitrogen fertility, and greenhouse gas emissions: Optimizing their joint economic management. Agric. Syst. 2019, 176, 102684. [CrossRef]

7. Cho, S.R.; Jeong, S.T.; Kim, G.Y.; Lee, J.G.; Kim, G.W. Evaluation of the carbon dioxide $\left(\mathrm{CO}_{2}\right)$ emission factor from lime ap-plied in temperate upland soil. Geoderma 2019, 337, 742-748. [CrossRef]

8. Kunhikrishnan, A.; Thangarajan, R.; Bolan, N.; Xu, Y.; Mandal, S.; Gleeson, D.; Seshadri, B.; Zaman, M.; Barton, L.; Tang, C.; et al. Functional relationships of soil acidification, liming, and greenhouse gas flux. Adv. Agron. 2016, 139, 1-71. [CrossRef]

9. Yagi, R.; Fidalski, J.; Tormena, C.A. The incorporation of limestone in consolidated no-tillage system reduces carbon stock in soil macroaggregates. Ciência Rural 2014, 44, 1962-1965. [CrossRef]

10. Jafer, D.G.; Hailu, G. Application of lime for acid soil amelioration and better soybean performance in South Western Ethiopia. J. Biol. Agric. Healthc. 2017, 7, 95-100. 
11. Gonzaga, M.I.S.; Mackowiak, C.; De Almeida, A.Q.; Junior, J.I.T.D.C.; Andrade, K.R. Positive and negative effects of biochar from coconut husks, orange bagasse and pine wood chips on maize (Zea mays L.) growth and nutrition. Catena 2018, 162, 414-420. [CrossRef]

12. Tomczyk, A.; Sokołowska, Z.; Boguta, P. Biochar physicochemical properties: Pyrolysis temperature and feedstock kind effects. Rev. Environ. Sci. BioTechnology 2020, 19, 191-215. [CrossRef]

13. Bhutto, A.W.; Qureshi, K.; Abro, R.; Harijan, K.; Zhao, Z.; Bazmi, A.A.; Abbas, T.; Yu, G. Progress in the production of biomass-toliquid biofuels to decarbonize the transport sector-Prospects and challenges. RSC Adv. 2016, 6, 32140-32170. [CrossRef]

14. Uslu, O.S.; Babur, E.; Alma, M.H.; Solaiman, Z.M. Walnut shell biochar increases seed germination and early growth of seedlings of fodder crops. Agriculture 2020, 10, 427. [CrossRef]

15. Sistani, K.R.; Simmons, J.R.; Jn-Baptiste, M.; Novak, J.M. Poultry litter, biochar, and fertilizer effect on corn yield, nutrient uptake, $\mathrm{N}_{2} \mathrm{O}$ and $\mathrm{CO}_{2}$ emissions. Environment 2019, 6, 55. [CrossRef]

16. Mosharrof, M.; Uddin, M.K.; Shamshuddin, J.; Sulaiman, M.F.; Shamsuzzaman, S.M.; Haque, A.N.A. Integrated use of bio-char and lime as a tool to improve maize yield and mitigate $\mathrm{CO}_{2}$ emission: A review. Chil. J. Agric. Res. 2021, 81, 1. [CrossRef]

17. Joseph, S.; Pow, D.; Dawson, K.; Rust, J.; Munroe, P.; Taherymoosavi, S.; Mitchell, D.R.; Robb, S.; Solaiman, Z.M. Biochar increases soil organic carbon, avocado yields and economic return over 4 years of cultivation. Sci. Total. Environ. 2020, $724,138153$. [CrossRef] [PubMed]

18. Ayodele, O.J.; Shittu, O.S. Fertilizer, lime and manure amendments for ultisols formed on coastal plain sands of Southern Nigeria. Agric. For. Fish. 2014, 3, 481-488. [CrossRef]

19. Wei, H.; Liu, Y.; Xiang, H.; Zhang, J.; Li, S.; Yang, J. Soil pH responses to simulated acid rain leaching in three agricultural soils. Sustainability 2019, 12, 280. [CrossRef]

20. Nweke, I.A. Influence of different leguminous crop on the ultisol that had been continuously cropped to cassava/maize for over six years. J. Soil Sci. Environ. Manag. 2016, 7, 222-229. [CrossRef]

21. Syuhada, A.; Shamshuddin, J.; Fauziah, C.; Rosenani, A.; Arifin, A. Biochar as soil amendment: Impact on chemical properties and corn nutrient uptake in a Podzol. Can. J. Soil Sci. 2016, 96, 400-412. [CrossRef]

22. Akingbola, O.O.; Adeyemo, A.J.; Oladele, S.O.; Ojeniyi, S.O. Physical status and infiltration dynamics of tropical alfisol of SouthWestern Nigeria as affected by poultry manure. Appl. Trop. Agric. 2016, 21, 102-111.

23. Schofield, H.K.; Pettitt, T.R.; Tappin, A.D.; Rollinson, G.K.; Fitzsimons, M.F. Biochar incorporation increased nitrogen and carbon retention in a waste-derived soil. Sci. Total. Environ. 2019, 690, 1228-1236. [CrossRef]

24. Hossain, Z.; Bahar, M.; Sarkar, B.; Donne, S.W.; Ok, Y.S.; Palansooriya, K.N.; Kirkham, M.B.; Chowdhury, S.; Bolan, N. Biochar and its importance on nutrient dynamics in soil and plant. Biochar 2020, 2, 379-420. [CrossRef]

25. Rabileh, M.A.; Shamshuddin, J.; Panhwar, Q.A.; Rosenani, A.B.; Anuar, A.R. Effects of biochar and/or dolomitic limestone application on the properties of Ultisol cropped to maize under glasshouse conditions. Can. J. Soil Sci. 2015, 95, 37-47. [CrossRef]

26. Ch'Ng, H.Y.; Haruna, A.O.; Majid, N.M.N.A.; Jalloh, M.B. Improving soil phosphorus availability and yield of Zea mays L. using biochar and compost derived from agro-industrial wastes. Ital. J. Agron. 2019, 14, 34-42. [CrossRef]

27. Solaiman, Z.M.; Shafi, M.I.; Beamont, E.; Anawar, H.M. Poultry litter biochar increases mycorrhizal colonisation, soil fertility and cucumber yield in a fertigation system on sandy soil. Agriculture 2020, 10, 480. [CrossRef]

28. Carvalho, M.L.; De Moraes, M.T.; Cerri, C.E.P.; Cherubin, M.R. Biochar amendment enhances water retention in a tropical sandy soil. Agriculture 2020, 10, 62. [CrossRef]

29. He, Y.; Zhou, X.; Jiang, L.; Li, M.; Du, Z.; Zhou, G.; Shao, J.; Wang, X.; Xu, Z.; Bai, S.H.; et al. Effects of biochar application on soil greenhouse gas fluxes: A meta-analysis. GCB Bioenergy 2016, 9, 743-755. [CrossRef]

30. Liu, S.; Zhang, Y.; Zong, Y.; Hu, Z.; Wu, S.; Zhou, J.; Jin, Y.; Zou, J. Response of soil carbon dioxide fluxes, soil organic carbon and microbial biomass carbon to biochar amendment: A meta-analysis. GCB Bioenergy 2015, 8, 392-406. [CrossRef]

31. Nguyen, D.H.; Scheer, C.; Rowlings, D.W.; Grace, P.R. Rice husk biochar and crop residue amendment in subtropical cropping soils: Effect on biomass production, nitrogen use efficiency and greenhouse gas emissions. Biol. Fertil. Soils 2015, 52, 261-270. [CrossRef]

32. Pedram, K. Genetic Potential of Selected Sweet Corn Inbred Lines and Analysis of Their Combining Ability Assisted by mi-Crosatellite DNA Markers. Ph.D. Thesis, Universiti Putra Malaysia, Selangor, Malaysia, 2012.

33. Benton, J.J. Laboratory Guide for Conducting Soil Tests and Plant Analysis; CRC Press: Boca Raton, FL, USA, 2001 ; ISBN 9780429132117.

34. Douglas, L.A.; Bremner, J.M. Extraction and colorimetric determination of urea in soils. Soil Sci. Soc. Am. J. 1970, 34, 859-862. [CrossRef]

35. Schollenberger, C.J.; Simon, R.H. Determination of exchange capacity and exchangeable bases in soil-Ammonium acetate method. Soil Sci. 1945, 59, 13-24. [CrossRef]

36. Bray, R.H.; Kurtz, L.T. Determination of total, organic, and available forms of phosphorus in soils. Soil Sci. 1945, 59, 39-46. [CrossRef]

37. Rimmer, D.L.; Rodwell, D.L. Soil Science: Methods and Application. J. Ecol. 1995, 83, 352. [CrossRef]

38. Tan, K.H. Soil and plant test. In Soil Sampling, Preparation, and Analysis, 2nd ed.; Tan, K.H., Ed.; CRC Press, Taylor \& Francis Group: Boca Raton, FL, USA, 2005; pp. 98-134.

39. Bouyoucos, G.J. Hydrometer method improved for making particle size analysis of soils. Agron. J. 1962, 54, 464-465. [CrossRef] 
40. Ahmedna, M.; Marshall, W.E.; Rao, R.M. Production of granular activated carbon from select agricultural by-products and evaluation of their physical, chemical, and adsorption properties. Bioresour. Technol. 1998, 71, 113-123. [CrossRef]

41. Cottenie, A. Soil testing and plant testing as a basis of fertilizer recommendation. FAO Soil Bull. 1980, 38, 70-73.

42. Song, W.; Guo, M. Quality variations of poultry litter biochar generated at different pyrolysis temperatures. J. Anal. Appl. Pyrol. 2012, 94, 138-145. [CrossRef]

43. Ashraf, M.; Waheed, A. Screening of local/exotic accessions of lentil (Lens culinaris Medic.) for salt tolerance at two growth stages. Plant Soil 1990, 128, 167-176. [CrossRef]

44. Hardy, F.; Lewis, A.H. A rapid electrometric method for measuring "lime requirements" of soils. J. Agric. Sci. 1929, 19, 17-25. [CrossRef]

45. Shen, Y.; Zhu, L.; Cheng, H.; Yue, S.; Li, S. Effects of biochar application on $\mathrm{CO}_{2}$ emissions from a cultivated soil under sem-iarid climate conditions in Northwest China. Sustainability 2017, 9, 1482. [CrossRef]

46. Haque, A.N.A.; Uddin, K.; Sulaiman, M.F.; Amin, A.M.; Hossain, M.; Zaibon, S.; Mosharrof, M. Assessing the increase in soil moisture storage capacity and nutrient enhancement of different organic amendments in paddy soil. Agriculture 2021, 11, 44. [CrossRef]

47. Masud, M.; Baquy, M.A.-A.; Akhter, S.; Sen, R.; Barman, A.; Khatun, M. Liming effects of poultry litter derived biochar on soil acidity amelioration and maize growth. Ecotoxicol. Environ. Saf. 2020, 202, 110865. [CrossRef]

48. Norazlina, A.B.; Fauziah, C.I.; Rosenani, A.B. Characterization of oil palm empty fruit bunch and rice husk biochar and their potential to adsorb arsenic and cadmium. Am. J. Agric. Biol. Sci. 2014, 9, 450-456. [CrossRef]

49. Zhang, K.; Chen, L.; Li, Y.; Brookes, P.C.; Xu, J.; Luo, Y. The effects of combinations of biochar, lime, and organic fertilizer on nitrification and nitrifiers. Biol. Fertil. Soils 2016, 53, 77-87. [CrossRef]

50. Gaskin, J.W.; Speir, R.A.; Harris, K.; Das, K.C.; Lee, R.D.; Morris, L.A.; Fisher, D.S. Effect of peanut hull and pine chip biochar on soil nutrients, corn nutrient status, and yield. Agron. J. 2010, 102, 623-633. [CrossRef]

51. Yuan, J.-H.; Xu, R.-K. The amelioration effects of low temperature biochar generated from nine crop residues on an acidic Ultisol. Soil Use Manag. 2010, 27, 110-115. [CrossRef]

52. Chintala, R.; Mollinedo, J.J.; Schumacher, T.E.; Malo, D.D.; Julson, J.L. Effect of biochar on chemical properties of acidic soil. Arch Agron. Soil Sci. 2013. [CrossRef]

53. Lehmann, J.; Gaunt, J.; Rondon, M. Bio-char sequestration in terrestrial ecosystems-A review. Mitig. Adapt. Strateg. Glob. Chang. 2006, 11, 403-427. [CrossRef]

54. Zhang, A.; Liu, Y.; Pan, G.; Hussain, Q.; Li, L.; Zheng, J.; Zhang, X. Effect of biochar amendment on maize yield and greenhouse gas emissions from a soil organic carbon poor calcareous loamy soil from Central China Plain. Plant Soil 2011, 351, $263-275$. [CrossRef]

55. Sukartono, U.W.H.; Utomo, W.H.; Kusuma, Z.; Nugroho, W.H. Soil fertility status, nutrient uptake, and maize (Zea mays L.) yield following biochar and cattle manure application on sandy soils of Lombok, Indonesia. J. Trop. Agric. 2011, 49, 47-52.

56. Trupiano, D.; Cocozza, C.; Baronti, S.; Amendola, C.; Vaccari, F.P.; Lustrato, G.; Di Lonardo, S.; Fantasma, F.; Tognetti, R.; Scippa, G.S. The effects of biochar and its combination with compost on lettuce (Lactuca sativa L.) growth, soil properties, and soil microbial activity and abundance. Int. J. Agron. 2017, 2017, 1-12. [CrossRef]

57. Zhang, A.; Bian, R.; Pan, G.; Cui, L.; Hussain, Q.; Li, L.; Zheng, J.; Zhang, X.; Han, X.; Yu, X. Effects of biochar amendment on soil quality, crop yield and greenhouse gas emission in a Chinese rice paddy: A field study of two consecutive rice growing cycles. Field Crops Res. 2012, 127, 153-160. [CrossRef]

58. Xie, Z.; Xu, Y.; Liu, G.; Liu, Q.; Zhu, J.; Tu, C.; Amonette, J.E.; Cadisch, G.; Yong, J.W.H.; Hu, S. Impact of biochar application on nitrogen nutrition of rice, greenhouse-gas emissions and soil organic carbon dynamics in two paddy soils of China. Plant Soil 2013, 370, 527-540. [CrossRef]

59. Knicker, M. Optimization of water using carbon-based adsorbents. Aust. J. Soil Res. 2007, 183, 249-255. [CrossRef]

60. Cao, X.; Harris, W. Properties of dairy-manure-derived biochar pertinent to its potential use in remediation. Bioresour. Technol. 2010, 101, 5222-5228. [CrossRef]

61. Koutcheiko, S.; Monreal, C.; Kodama, H.; McCracken, T.; Kotlyar, L. Preparation and characterization of activated carbon derived from the thermo-chemical conversion of chicken manure. Bioresour. Technol. 2007, 98, 2459-2464. [CrossRef]

62. $\mathrm{Ch}$ 'Ng, H.Y.; Ahmed, O.H.; Majid, N.M.A. Improving phosphorus availability in an acid soil using organic amendments produced from agroindustrial wastes. Sci. World J. 2014, 2014, 1-6. [CrossRef]

63. Madiba, O.F.; Solaiman, Z.M.; Carson, J.K.; Murphy, D.V. Biochar increases availability and uptake of phosphorus to wheat under leaching con-ditions. Biol. Fertil. Soils 2016, 52, 439-446. [CrossRef]

64. Marsi, M.; Sabaruddin, S. Phosphate adsorption capacity and organic matter effect on dynamics of P availability in upland ultisol and lowland inceptisol. J. Trop. Soils 2011, 16, 107-114. [CrossRef]

65. Panhwar, Q.A.; Naher, U.A.; Shamshuddin, J.; Ismail, M.R. Effects of biochar and ground magnesium limestone application, with or without bio-fertilizer addition, on biochemical properties of an acid sulfate soil and rice yield. Agronomy 2020, 10, 1100. [CrossRef]

66. Kloss, S.; Zehetner, F.; Dellantonio, A.; Hamid, R.; Ottner, F.; Liedtke, V.; Schwanninger, M.; Gerzabek, M.H.; Soja, G. Characterization of slow pyrolysis biochars: Effects of feed stocks and pyrolysis temperature on biochar properties. J. Environ. Qual. 2012, 41, 990-1000. [CrossRef] 
67. Mensah, A.K.; Frimpong, K.A. Biochar and/or compost applications improve soil properties, growth, and yield of maize grown in acidic rainforest and coastal savannah soils in Ghana. Int. J. Agron. 2018, 6837404. [CrossRef]

68. Zaidun, S.W.; Jalloh, M.B.; Awang, A.; Sam, L.M.; Besar, N.A.; Musta, B.; Ahmed, O.H.; Omar, L. Biochar and clinoptilolite zeolite on selected chemical properties of soil cultivated with maize (Zea mays L.). Euras. J. Soil Sci. EJSS 2019, 8, 1-10. [CrossRef]

69. Gautam, D.K.; Bajracharya, R.M.; Sitaula, B.K. Effects of biochar and farm yard manure on soil properties and crop growth in an agroforestry system in the Himalaya. Sustain. Agric. Res. 2017, 6, 74. [CrossRef]

70. Nigussie, A.; Kissi, E.; Misaganaw, M.; Ambaw, G. Effects of biochar application on soil properties and nutrient uptake of lettuces (Lactuca sativa) grown in polluted soils. Am. Euras. J. Agric. Environ. Sci. 2012, 12, 369-376.

71. Laird, D.A.; Fleming, P.; Davis, D.D.; Horton, R.; Wang, B.; Karlen, D.L. Impact of biochar amendments on the quality of a typical Midwestern agricultural soil. Geoderma 2010, 158, 443-449. [CrossRef]

72. Wang, L.; Xue, C.; Nie, X.; Liu, Y.; Chen, F. Effects of biochar application on soil potassium dynamics and crop uptake. J. Plant Nutr. Soil Sci. 2018, 181, 635-643. [CrossRef]

73. Abewa, A.; Yitaferu, B.; Selassie, Y.G.; Amare, T.T. The role of biochar on acid soil reclamation and yield of teff (Eragrostis tef [Zucc] Trotter) in Northwestern Ethiopia. J. Agric. Sci. 2013, 6, 1. [CrossRef]

74. Zhu, Q.-H.; Peng, X.-H.; Huang, T.-Q.; Xie, Z.-B.; Holden, N. Effect of biochar addition on maize growth and nitrogen use efficiency in acidic red soils. Pedosphere 2014, 24, 699-708. [CrossRef]

75. Qian, L.; Chen, B.; Hu, D. Effective alleviation of aluminum phytotoxicity by manure-derived biochar. Environ. Sci. Technol. 2013, 47, 2737-2745. [CrossRef] [PubMed]

76. Sasmita, K.D.; Iswandi, A.; Syaiful, A.; Sudirman, Y.; Gunawan, D. Application of biochar and organic fertilizer on acid soil as growing medium for Cacao (Theobroma cacao L.) seedlings. Int. J. Sci. Basic Appl. Res. 2017, 36, 261-273.

77. Masulili, A.; Sudarso, J.K.Y.; Utomo, W.H.; Veteran, J.; Syechfani, M.S. Rice husk biochar for rice-based cropping system in acid soil. The characteristics of rice husk biochar and its influence on the properties of acid sulfate soils and rice growth in West Kalimantan, Indonesia. J. Agric. Sci. 2010, 2, 39-47. [CrossRef]

78. Lentz, R.D.; Ippolito, J.A. Biochar and manure affect calcareous soil and corn silage nutrient concentrations and uptake. J. Environ. Qual. 2011, 41, 1033-1043. [CrossRef]

79. Devika, O.S.; Prasad, P.R.; Rani, P.P.; Pathy, R.L. Nutrient status of soils influenced by the interaction of Biochar and FYM with chemical Fertiizers. J. Pharm. Innov. 2018, 7, 174-177.

80. Ullah, Z.; Jamali, A.Z.; Ali, M.; Khan, B.; Yousaf, S.; Ziad, T. Effects of biochar on soil chemical properties in relation at different intervals. J. Bio. Environ. Sci. 2018, 12, 272-277.

81. Amonette, J.E.; Joseph, S. Characteristics of biochar: Microchemical properties. In Biochar for Environmental Management: Science and Technology, 1st ed.; Lehmann, J., Joseph, S., Eds.; Earthscan: London, UK, 2009; pp. 33-52.

82. Mohan, D.; Abhishek, K.; Sarswat, A.; Patel, M.; Singh, P.; Pittman, C.U. Biochar production and applications in soil fertility and carbon sequestration-A sustainable solution to crop-residue burning in India. RSC Adv. 2018, 8, 508-520. [CrossRef]

83. Fuentes, J.P.; Bezdicek, D.F.; Flury, M.; Albrecht, S.; Smith, J.L. Microbial activity affected by lime in a long-term no-till soil. Soil Tillage Res. 2006, 88, 123-131. [CrossRef]

84. Sarfaraz, Q.; Silva, L.; Drescher, G.; Zafar, M.; Severo, F.; Kokkonen, A.; Molin, G.; Shafi, M.I.; Shafique, Q.; Solaiman, Z. Characterization and carbon mineralization of biochars produced from different animal manures and plant residues. Sci. Rep. 2020, 10, 955-959. [CrossRef]

85. Bramble, D.S.E.; Gouveia, G.A.; Ramnarine, R. Organic residues and ammonium effects on $\mathrm{CO}_{2}$ emissions and soil quality indicators in limed acid tropical soils. Soil Syst. 2019, 3, 16. [CrossRef]

86. Fidel, R.B.; Laird, D.A.; Parkin, T.B. Effect of biochar on soil greenhouse gas emissions at the laboratory and field scales. Soil Syst. 2019, 3, 8. [CrossRef]

87. Oo, A.Z.; Sudo, S.; Akiyama, H.; Win, K.T.; Shibata, A.; Yamamoto, A.; Sano, T.; Hirono, Y. Effect of dolomite and biochar addition on $\mathrm{N}_{2} \mathrm{O}$ and $\mathrm{CO}_{2}$ emissions from acidic tea field soil. PLoS ONE 2018, 13, e0192235. [CrossRef]

88. Moreno-Cornejo, J.; Zornoza, R.; Faz, A. Carbon and nitrogen mineralization during decomposition of crop residues in a calcareous soil. Geoderma 2014, 230-231, 58-63. [CrossRef]

89. El-Naggar, A.H.; Usman, A.R.; Al-Omran, A.; Ok, Y.S.; Ahmad, M.; Al-Wabel, M.I. Carbon mineralization and nutrient availability in calcareous sandy soils amended with woody waste biochar. Chemosphere 2015, 138, 67-73. [CrossRef] [PubMed]

90. Bruun, E.W.; Hauggaard-Nielsen, H.; Ibrahim, N.; Egsgaard, H.; Ambus, P.; Jensen, P.A.; Dam-Johansen, K. Influence of fast pyrolysis temperature on biochar labile fraction and short-term carbon loss in a loamy soil. Biomass Bioenergy 2011, 35, 1182-1189. [CrossRef]

91. Rochette, P.; Angers, D.A.; Chantigny, M.H.; Gagnon, B.; Bertrand, N. In situ mineralization of dairy cattle manure as de-termined using soil-surface carbon dioxide fluxes. Soil Sci. Soc. Am. J. 2006, 70, 744-752. [CrossRef]

92. Kong, Y.; Watanabe, M.; Nagano, H.; Watanabe, K.; Yashima, M.; Inubushi, K. Effects of land-use type and nitrogen addition on nitrous oxide and carbon dioxide production potentials in Japanese Andosols. Soil Sci. Plant Nutr. 2013, 59, 790-799. [CrossRef]

93. Wang, J.; Pan, X.; Liu, Y.; Zhang, X.; Xiong, Z. Effects of biochar amendment in two soils on greenhouse gas emissions and crop production. Plant Soil 2012, 360, 287-298. [CrossRef]

94. Zimmerman, A.R.; Gao, B.; Ahn, M.Y. Positive and negative carbon mineralization priming effects among a variety of bio-charamended soils. Soil Biol. Biochem. 2011, 43, 1169-1179. [CrossRef] 
95. Smith, J.L.; Collins, H.P.; Bailey, V.L. The effect of young biochar on soil respiration. Soil Biol. Biochem. 2010, 42, $2345-2347$. [CrossRef]

96. Chenfei, L.; Li, S.; Liang, C.; Xu, Q.; Li, Y.; Qin, H.; Fuhrmann, J.J. Response of microbial community structure and function to short-term biochar amendment in an intensively managed bamboo (Phyllostachys praecox) plantation soil: Effect of particle size and addition rate. Sci. Total. Environ. 2017, 574, 24-33. [CrossRef]

97. Lehmann, J.; Rillig, M.C.; Thies, J.; Masiello, C.A.; Hockaday, W.C.; Crowley, D. Biochar effects on soil biota-A review. Soil Biol. Biochem. 2011, 43, 1812-1836. [CrossRef]

98. Cross, A.; Sohi, S.P. The priming potential of biochar products in relation to labile carbon contents and soil organic matter status. Soil Biol. Biochem. 2011, 43, 2127-2134. [CrossRef]

99. Chen, G.; Wang, X.; Zhang, R. Decomposition temperature sensitivity of biochars with different stabilities affected by organic carbon fractions and soil microbes. Soil Tillage Res. 2019, 186, 322-332. [CrossRef]

100. Sui, Y.; Gao, J.; Liu, C.; Zhang, W.; Lan, Y.; Li, S.; Meng, J.; Xu, Z.; Tang, L. Interactive effects of straw-derived biochar and N fertilization on soil C storage and rice productivity in rice paddies of Northeast China. Sci. Total Environ. 2016, 544, 203-210. [CrossRef]

101. Spokas, K.A.; Novak, J.M.; Stewart, C.E.; Cantrell, K.B.; Uchimiya, M.; DuSaire, M.G.; Ro, K.S. Qualitative analysis of volatile organic compounds on biochar. Chemosphere 2011, 85, 869-882. [CrossRef] 